\title{
SPIN POLYNOMIAL FUNCTORS AND REPRESENTATIONS OF SCHUR SUPERALGEBRAS
}

\author{
JONATHAN AXTELL
}

\begin{abstract}
We introduce categories of homogeneous strict polynomial functors, Pol ${ }_{d, \mathbb{k}}^{\mathrm{I}}$ and Pol ${ }_{d, \mathbb{k}}^{\mathrm{II}}$, defined on vector superspaces over a field $\mathbb{k}$ of characteristic not equal 2 . These categories are related to polynomial representations of the supergroups $G L(m \mid n)$ and $Q(n)$. In particular, we prove an equivalence between $\mathrm{Pol}_{d, \mathbb{k}}^{\mathrm{I}}, \mathrm{Pol}_{d, \mathbb{k}}^{\mathrm{II}}$ and the category of finite dimensional supermodules over the Schur superalgebra $\mathcal{S}(m \mid n, d), \mathcal{Q}(n, d)$ respectively provided $m, n \geq d$. We also discuss some aspects of Sergeev duality from the viewpoint of the category Pol $_{d, \mathbb{k}}^{\mathrm{II}}$.
\end{abstract}

\section{INTRODUCTION}

Strict polynomial functors were introduced by Friedlander and Suslin in [FS] as a tool for use in their investigation of rational cohomology of finite group schemes over a field. Let us briefly recall the definition.

Suppose $\mathbb{k}$ is an arbitrary field, and let $v c_{\mathbb{k}}$ denote the category of finite dimensional $\mathbb{k}$-vector spaces. Also, let $\mathfrak{s c h}_{\mathbb{k}}$ be the category of all schemes over $\mathbb{k}$. Then, by identifying each hom-space with its associated affine scheme, we obtain an $\mathfrak{s c h} \mathfrak{h}_{\mathbb{k}}$-enriched category $\mathfrak{v e c}_{\mathbb{k}}$ (in the sense of $\left[\mathrm{Ke}\right.$ ) with the same objects as $\operatorname{vec}_{\mathbb{k}}$. Although stated somewhat differently in [FS, Definition 2.1], a strict polynomial functor may be defined as an $\mathfrak{s c h}_{\mathbb{k}^{\mathbb{k}}}$-enriched functor from $\mathfrak{v} \mathfrak{c}_{\mathbb{k}}$ to itself. From this perspective, it is clear that a strict polynomial functor $T$ yields, by evaluation at any $V \in \mathrm{vec}_{\mathbb{k}}$, a polynomial representation $T(V)$ of the affine group scheme $G L(V)$. Let us denote by $\mathfrak{p o l}_{d}(G L(V))$ the category of finite dimensional polynomial representations of $G L(V)$ which are homogeneous of degree $d$. Then a strict polynomial functor $T$ is said to be homogeneous of degree $d$ if $T(V) \in \mathfrak{p o l}_{d}(G L(V))$ for all $V \in \mathfrak{v e c}_{\mathrm{k}}$. We denote by $\mathcal{P}_{d}$ the category of all such homogeneous strict polynomial functors. The morphisms in $\mathcal{P}_{d}$ are $\mathfrak{s c h}_{\mathbb{k}}$-enriched natural transformations.

Assume that $n \geq d$. Then, evaluation at $V=\mathbb{k}^{n}$ in fact gives an equivalence of categories

$$
\mathcal{P}_{d} \stackrel{\sim}{\longrightarrow} \operatorname{pol}_{d}\left(G L_{n}\right) .
$$

This follows from the definition of the Schur algebra $S(n, d)$ in terms of the coordinate ring of $G L_{n}$ (as in Green's monograph [G]) and [FS, Theorem 3.2], which provides an equivalence between $\mathcal{P}_{d}$ and the category of finite dimensional modules over $S(n, d)$. We remark that there is an alternate definition of the category

Received by the editors February 8, 2013 and, in revised form, May 28, 2013.

2010 Mathematics Subject Classification. Primary 16D90, 17A70, 18D20, 20G05, 20G43; Secondary $14 \mathrm{~L} 15$.

This work was supported by the BRL research fund grant \#2013055408 of the National Research Foundation of Korea. 
$\mathcal{P}_{d}$ which makes the relationship with $S(n, d)$-modules more transparent (see e.g. $\underline{\mathrm{Kr}}, \mathrm{P}$ ). In this new definition, $\mathfrak{s c h}_{\mathbb{k}}$-enriched functors are replaced by $\mathbb{k}$-linear functors defined on a category of divided powers.

The aim of this paper is to provide an analogue of [FS, Theorem 3.2] for Schur superalgebras. More specifically, suppose now that $\mathbb{k}$ is a field of characteristic $p \neq 2$. In this context, the Schur superalgebras $\mathcal{S}(m \mid n, d)$ and $\mathcal{Q}(n, d)$ were studied by Donkin [D] and Brundan and Kleshchev BrK1, respectively. In both works there was obtained a classification of finite dimensional irreducible supermodules over the corresponding Schur superalgebra. (In BrK1] the field $\mathbb{k}$ is assumed to be algebraically closed.) In this paper, we introduce categories of strict polynomial functors defined on vector superspaces, and we show that each such category is equivalent to the category of finite dimensional supermodules over one of the above Schur superalgebras. To define strict polynomial functors on superspaces, it is more convenient for us to follow the approach involving categories of divided powers. In the last section, however, we provide a definition of strict polynomial functors as "enriched functors" which is closer to Friedlander and Suslin's original definition.

The content of the paper is as follows. In Section 2, we give necessary preliminary results concerning superalgebras and supermodules. In Section 3, we introduce the categories $\mathrm{Pol}_{d}^{(\dagger)}=\mathrm{Pol}_{d, \mathbb{k}}^{(\dagger)}(\dagger=\mathrm{I}, \mathrm{II})$ of homogeneous strict polynomial functors, whose objects are $\mathbb{k}$-linear functors defined on categories of vector superspaces. We also discuss some of the usual facets of polynomial functors such as Kuhn duality and Yoneda's lemma in this new context. (See $[\mathrm{Kr}, \mathrm{P}, \mathrm{T} 2$ for descriptions of the corresponding classical notions).

In Section 4, we prove our main result, Theorem 4.2, which gives an equivalence between $\mathrm{Pol}_{d}^{(\mathrm{I})}, \mathrm{Pol}_{d}^{(\mathrm{II})}$ and the category of finite dimensional supermodules over $\mathcal{S}(m \mid n, d), \mathcal{Q}(n, d)$ respectively for $m, n \geq d$. We are then able to obtain a classification of simple objects in both categories using the classifications of [D] and BrK1. As another application of Theorem 4.2, we give an exact functor from the category $\mathrm{Pol}_{d}^{(\mathrm{II})}$ to the category of finite dimensional left supermodules over the Sergeev superalgebra $\mathcal{W}(d)$. This functor may be viewed as a categorical analogue of Sergeev duality, as described by Sergeev in Ser when $p=0$ and by Brundan and Kleshchev BrK1 in arbitrary characteristic $p \neq 2$. Since the representation theory of $\mathcal{W}(d)$ is closely related to that of the spin symmetric group algebra $\mathbb{k}_{d}^{-}$ (cf. BrK1]), we may refer to objects of $\mathrm{Pol}_{d}^{(\mathrm{II})}$ as spin polynomial functors.

In Section 5, we conclude by describing categories $\mathfrak{P o r}_{d}^{(\mathrm{I})}$ and $\mathfrak{P o r}_{d}^{(\mathrm{II})}$ consisting of homogeneous $\mathfrak{s s c h} \mathfrak{\mathbb { K }}_{\mathbb{k}}$-enriched functors, where $\mathfrak{s i c h} \mathfrak{\mathbb { K }}_{\mathbb{k}}$ denotes the category of all superschems over $\mathbb{k}$. This definition may be viewed as a "super analogue" of Friendlander and Suslin's original definition of strict polynomial functors. In Theorem 5.4 we show that our two definitions of strict polynomial functors are equivalent. One of the benefits of the classical approach is that the relationship between strict polynomial functors and polynomial representations of the supergroups $G L(m \mid n)$ and $Q(n)$ appears naturally from the definition of $\mathfrak{s s c h} \mathfrak{l}_{\mathbb{k}}$-enriched functors.

Finally, let us mention our original motivation for considering categories of polynomial functors defined on vector superspaces. In HTY], J. Hong, A. Touzé and O. Yacobi showed that the category of all strict polynomial functors of finite degree

$$
\mathcal{P}=\bigoplus_{d \geq 0} \mathcal{P}_{d}
$$


defined over an infinite field $\mathbb{k}$ of characteristic $p$, provides a categorification of level 1 Fock space representations (in the sense of Chuang and Rouquier) for an affine Kac-Moody algebra $\mathfrak{g}$ of type $A_{\infty}$ (if $p=0$ ) or of type $A_{p-1}^{(1)}$ (in case $p>0$ ). We conjecture that the category of all spin polynomial functors

$$
\mathrm{Pol}_{\mathbb{k}}^{(\mathrm{II})}=\bigoplus_{d \geq 0} \mathrm{Pol}_{d, \mathbb{k}}^{(\mathrm{II})}
$$

defined over an algebraically closed field $\mathbb{k}$ of characteristic $p \neq 2$, provides a categorification of certain level 1 Fock spaces for an affine Kac-Moody algebra of type $B_{\infty}($ if $p=0)$ or of type $A_{p-1}^{(2)}($ if $p>2)$.

\section{Superalgebras AND SUPERMOdules}

In this section, we give preliminary results on superalgebras and supermodules needed for the remainder. See [BrK1, K, Ch.12-13], L, Ch.1] and [Man, Ch.3] for more details. Although our notation sometimes differs from these references.

2.1. Preliminaries. Let us fix a field $\mathbb{k}$, which we assume is of characteristic $p \neq 2$. A vector superspace is a $\mathbb{Z}_{2}$-graded $\mathbb{k}$-vector space $M=M_{0} \oplus M_{1}$. We denote the degree of a homogeneous vector, $v \in M$, by $|v| \in \mathbb{Z}_{2}$. A subsuperspace of $M$ is a subspace $N$ of $M$ such that $N=\left(N \cap M_{0}\right) \oplus\left(N \cap M_{1}\right)$. We let $\bar{M}$ denote the underlying ordinary vector space of a given superspace $M$, and we write $\operatorname{sdim}(M)=(m, n)$ if $\operatorname{dim}\left(\overline{M_{0}}\right)=m$ and $\operatorname{dim}\left(\overline{M_{1}}\right)=n$.

Given a pair of vector superspaces $M, N$ we view the direct sum $M \oplus N$ and the tensor product $M \otimes N$ as vector superspaces by setting: $(M \oplus N)_{i}=M_{i} \oplus N_{i}$ $\left(i \in \mathbb{Z}_{2}\right),(M \otimes N)_{0}=M_{0} \otimes N_{0} \oplus M_{1} \otimes N_{1}$ and $(M \otimes N)_{1}=M_{0} \otimes N_{1} \oplus M_{1} \otimes N_{0}$. We also consider the vector space $\operatorname{Hom}(M, N)=\operatorname{Hom}_{\mathbb{k}}(M, N)$ of all $\mathbb{k}$-linear maps of $M$ into $N$ as a superspace by letting $\operatorname{Hom}(M, N)_{i}$ consist of the homogeneous maps of degree $i$ for $i \in \mathbb{Z}_{2}$, i.e., the maps $f: M \rightarrow N$ such that $f\left(M_{j}\right) \subseteq N_{i+j}$ for $j \in \mathbb{Z}_{2}$. The elements of $\operatorname{Hom}(M, N)_{0}$ are called even linear maps, and the elements of $\operatorname{Hom}(M, N)_{1}$ are called odd. The $\mathbb{k}$-linear dual $M^{\vee}=\operatorname{Hom}(M, \mathbb{k})$ is a superspace by viewing $\mathbb{k}$ as vector superspace concentrated in degree 0 . Let $\mathrm{svec}_{\mathbb{k}}$ denote the category of all finite dimensional $\mathbb{k}$-vector superspaces with arbitrary linear maps as morphisms.

If $M \in$ svec $_{\mathbb{k}}$, then for $f \in M^{\vee}$ and $v \in M$, we write

$$
\langle f, v\rangle=f(v) \in \mathbb{k}
$$

to denote the pairing between $M$ and $M^{\vee}$. We identify $M$ with $\left(M^{\vee}\right)^{\vee}$ as superspaces by setting

$$
\langle v, f\rangle=(-1)^{|v||f|}\langle f, v\rangle
$$

for $v \in M, f \in M^{\vee}$.

A superalgebra is a superspace $\mathcal{A}$ with the additional structure of an associative unital $\mathbb{k}$-algebra such that $\mathcal{A}_{i} \mathcal{A}_{j} \subseteq \mathcal{A}_{i+j}$ for $i, j \in \mathbb{Z}_{2}$. By forgetting the grading we may consider any superalgebra $\mathcal{A}$ as an ordinary algebra, denoted by $\overline{\mathcal{A}}$. A superalgebra homomorphism $\vartheta: \mathcal{A} \rightarrow \mathcal{B}$ is an even linear map that is an algebra homomorphism in the usual sense; its kernel is a superideal, i.e., an ordinary twosided ideal which is also a subsuperspace. An antiautomorphism $\tau: \mathcal{A} \rightarrow \mathcal{A}$ of a superalgebra $\mathcal{A}$ is an even linear map which satisfies $\tau(a b)=\tau(b) \tau(a)$. 
Given two superalgebras $\mathcal{A}$ and $\mathcal{B}$, we view the tensor product of superspaces $\mathcal{A} \otimes \mathcal{B}$ as a superalgebra with multiplication defined by

$$
(a \otimes b)\left(a^{\prime} \otimes b^{\prime}\right)=(-1)^{|b|\left|a^{\prime}\right|}\left(a a^{\prime}\right) \otimes\left(b b^{\prime}\right) \quad\left(a, a^{\prime} \in \mathcal{A}, b, b^{\prime} \in \mathcal{B}\right) .
$$

We note that $\mathcal{A} \otimes \mathcal{B} \cong \mathcal{B} \otimes \mathcal{A}$, an isomorphism being given by

$$
a \otimes b \mapsto(-1)^{|a||b|} b \otimes a \quad(a \in \mathcal{A}, b \in \mathcal{B}) .
$$

2.2. Tensor powers. Let $M$ be a vector superspace. The tensor superalgebra $T^{*} M$ is the tensor algebra

$$
T^{*} M=\bigoplus_{d \geq 0} M^{\otimes d}
$$

regarded as a vector superspace. It is the free associative ( $\mathbb{Z}$-graded) superalgebra generated by $M$.

The symmetric superalgebra $S^{*} M$ is the quotient of $T^{*} M$ by the super ideal

$$
\mathcal{I}=\left\langle x \otimes y-(-1)^{|x||y|} y \otimes x ; x, y \in M\right\rangle .
$$

Since $\mathcal{I}$ is a $\mathbb{Z}$-graded homogeneous ideal, there exists a gradation $S^{*} M=\bigoplus_{d \geq 0} S^{d} M$. Now we may view the ordinary symmetric algebra $S y m^{*} \overline{M_{0}}$ as a superspace concentrated in degree zero. We may also view the ordinary exterior algebra $\Lambda^{*} \overline{M_{1}}$ as a superspace by reducing its $\mathbb{Z}$-grading $\bmod 2 \mathbb{Z}$. In this way both $S y m^{*} \overline{M_{0}}$ and $\Lambda^{*} \overline{M_{1}}$ may be regarded as $\mathbb{Z}$-graded superalgebras. One may check that we have a $\mathbb{Z}$-graded superalgebra isomorphism:

$$
S^{*} M \cong S y m^{*} \overline{M_{0}} \otimes \Lambda^{*} \overline{M_{1}} .
$$

A superalgebra $\mathcal{A}$ is called commutative if $a b=(-1)^{|a||b|} b a$ for all $a, b \in \mathcal{A}$. The superalgebra $S^{*} M$ is the free commutative ( $\mathbb{Z}$-graded) superalgebra generated by $M$.

2.3. Divided powers. Suppose $d \in \mathbb{Z}_{\geq 1}$. There is a unique (even) right action of the symmetric group $\mathfrak{S}_{d}$ on the tensor power $M^{\otimes d}$ such that each transposition $(i i+1)$ for $1 \leq i \leq d-1$ acts by:

$$
\left(v_{1} \otimes \cdots \otimes v_{d}\right) \cdot(i i+1)=(-1)^{\left|v_{i}\right|\left|v_{i+1}\right|} v_{1} \otimes \cdots \otimes v_{i+1} \otimes v_{i} \otimes \cdots \otimes v_{d},
$$

for any $v_{1}, \ldots, v_{d} \in M$ with $v_{i}, v_{i+1}$ being $\mathbb{Z}_{2}$-homogeneous. Denote the invariant subsuperspace of this action by

$$
\Gamma^{d} M:=\left(M^{\otimes d}\right)^{\mathfrak{S}_{d}},
$$

and let $\Gamma^{0} M=\mathbb{k}$. Now the symmetric power is the coinvariant superspace $S^{d} M=$ $\left(M^{\otimes d}\right)_{\mathfrak{S}_{d}}$. Hence, given arbitrary vector superspaces $V, W$ there are natural even isomorphisms,

$$
\operatorname{Hom}_{\mathfrak{S}_{d}}\left(V, M^{\otimes d}\right) \cong \operatorname{Hom}\left(V, \Gamma^{d} M\right), \operatorname{Hom}_{\mathfrak{S}_{d}}\left(M^{\otimes d}, W\right) \cong \operatorname{Hom}\left(S^{d} M, W\right),
$$

where $V$ and $W$ are considered as trivial $\mathfrak{S}_{d}$-modules. There is also a right action of $\mathfrak{S}_{d}$ on $\left(M^{\otimes d}\right)^{\vee}$ given by $(f . \sigma)(v)=f\left(v \cdot \sigma^{-1}\right)$, for $f \in\left(M^{\otimes d}\right)^{\vee}, v \in M^{\otimes d}$ and $\sigma \in \mathfrak{S}_{d}$. Furthermore, there is a natural even isomorphism

$$
\Gamma^{d}(M)^{\vee} \cong S^{d}\left(M^{\vee}\right) \text {. }
$$


Now let $\Gamma^{*} M$ be the $\mathbb{Z}$-graded superspace $\bigoplus_{d \geq 0} \Gamma^{d} M$. For $d, e \in \mathbb{Z}_{\geq 0}$ define an even bilinear map $\Gamma^{d} M \otimes \Gamma^{e} M \rightarrow \Gamma^{d+e} M$ by setting

$$
f \cdot g:=\sum_{\sigma \in \mathfrak{S}_{d+e} / \mathfrak{S}_{d} \times \mathfrak{S}_{e}}(f \otimes g) . \sigma,
$$

for $f \in \Gamma^{d} M, g \in \Gamma^{e} M$. It can be checked that these maps make $\Gamma^{*}(M)$ into a (Z्Z-graded) superalgebra, called the divided power superalgebra.

Now let $D^{*}\left(\overline{M_{0}}\right)$ denote the ordinary divided powers algebra of the vector space $\overline{M_{0}}$ (cf. $\mathrm{B}$ ). Viewed as a vector superspace concentrated in degree zero, $D^{*}\left(\overline{M_{0}}\right)$ is a $\mathbb{Z}$-graded superalgebra. Also note that we have a natural embedding of superspaces: $\Lambda^{d}\left(\overline{M_{1}}\right) \hookrightarrow\left(M_{1}\right)^{\otimes d}$. We then have even isomorphisms of $\mathbb{Z}$-graded superspaces

$$
\Gamma^{*} M_{0} \cong D^{*}\left(\overline{M_{0}}\right), \quad \Gamma^{*} M_{1} \cong \Lambda^{*}\left(\overline{M_{1}}\right) .
$$

Given $f \in \Gamma^{*} M_{0}, g \in \Gamma^{*} M_{1}$, let $\bar{f} \in D^{*}\left(\overline{M_{0}}\right), \bar{g} \in \Lambda^{*}\left(\overline{M_{1}}\right)$ denote the corresponding images with respect to the above isomorphism. Then there is an isomorphism of $\mathbb{Z}$-graded superalgebras

$$
D^{*}\left(\overline{M_{0}}\right) \otimes \Lambda^{*}\left(\overline{M_{1}}\right) \cong \Gamma^{*} M
$$

given by mapping $\bar{f} \otimes \bar{g} \mapsto f \cdot g$.

2.4. Supermodules. Let $\mathcal{A}$ be a superalgebra. A left $\mathcal{A}$-supermodule is a superspace $V$ which is a left $\mathcal{A}$-module in the usual sense, such that $\mathcal{A}_{i} V_{j} \subseteq \mathcal{A}_{i+j}$ for $i, j \in \mathbb{Z}_{2}$. One may similarly define right $\mathcal{A}$-supermodules. A homomorphism $\varphi: V \rightarrow W$ of left $\mathcal{A}$-supermodules $V$ and $W$ is a (not necessarily homogeneous) linear map such that

$$
\varphi(a v)=(-1)^{|\varphi||a|} a \varphi(v) \quad(a \in \mathcal{A}, v \in V) .
$$

We denote by $\mathcal{A}_{\text {smod }}$ the category of finite dimensional left $\mathcal{A}$-supermodules with $\mathcal{A}$-homomorhpisms. A homomorphism, $\varphi: V \rightarrow W$, of right $\mathcal{A}$-supermodules $V$ and $W$ is a (not necessarily homogeneous) linear map such that

$$
\varphi(v a)=\varphi(v) a \quad(a \in \mathcal{A}, v \in V) .
$$

Let $\operatorname{smod}_{\mathcal{A}}$ denote the category of finite dimensional right $\mathcal{A}$-supermodules with $\mathcal{A}$-homomorphisms.

2.5. Parity change functor. Suppose $V$ is a left or right $\mathcal{A}$-supermodule. Then define a new supermodule $\Pi V$ which is the same vector space as $V$ but with opposite $\mathbb{Z}_{2}$-grading. For right supermodules, the new right action is the same as in $V$. The action on morphisms is given by $\Pi f=(-1)^{|f|} f$. For left supermodules, the new left action of $a \in \mathcal{A}$ on $v \in \Pi V$ is defined in terms of the old one by $a \cdot v:=(-1)^{|a|} a v$. On a morphism $f, \Pi f$ is the same underlying linear map as $f$. Let us write $\mathbb{k}^{m \mid n}=\mathbb{k}^{m} \oplus(\Pi \mathbb{k})^{n}$.

Examples 2.1. We have the following examples of finite dimensional associative superalgebras.

(i) If $M$ is a superspace, then $\operatorname{End}(M)=\operatorname{Hom}_{\mathbb{k}}(M, M)$ is a superalgebra. In particular, we write $\mathcal{M}_{m, n}=\operatorname{End}\left(\mathbb{k}^{m \mid n}\right)$. 
(ii) Let $V \in \mathrm{svec}_{\mathbb{k}}$, and suppose $J$ is a degree one involution in $\operatorname{End}(V)$. This is possible if and only if $\operatorname{dim} \overline{V_{0}}=\operatorname{dim} \overline{V_{1}}$. Let us consider the superalgebra

$$
\mathcal{Q}(V, J)=\left\{\varphi \in \operatorname{End}(V) ; \varphi J=(-1)^{|\varphi|} J \varphi\right\} .
$$

Suppose that $\operatorname{sdim} V=(n, n)$, and let $\left\{v_{1}, \ldots, v_{n}\right\}$ (resp. $\left.\left\{v_{1}^{\prime}, \ldots, v_{n}^{\prime}\right\}\right)$ a basis of $V_{0}$ (resp. $\left.V_{1}\right)$. Let $J_{V}$ be the unique involution in $\operatorname{End}_{\mathbb{k}}(V)$ such that $J v_{i}=v_{i}^{\prime}$ for $1 \leq i \leq n$. Then we may write elements of $\mathcal{Q}\left(V, J_{V}\right)$ with respect to the basis $\left\{v_{1}, \ldots, v_{n}, v_{1}^{\prime}, \ldots, v_{n}^{\prime}\right\}$ as matrices of the form

$$
\left(\begin{array}{cc}
A & B \\
-B & A
\end{array}\right)
$$

where $A, B$ are $n \times n$ matrices, with $A=0$ for odd endomorphisms and $B=0$ for even ones.

Suppose that $\mathbb{k}$ is algebraically closed. Recall (cf. [K, ch.12]) that all odd involutions $J \in \operatorname{End}(V)$ are then mutually conjugate (by an invertible element of $\left.\operatorname{End}(V)_{0}\right)$. Hence, any superalgebra $\mathcal{Q}(V, J)$ is isomorphic to the superalgebra $\mathcal{Q}_{n}$, consisting of all matrices of the form (5).

(iii) The Clifford superalgebra, $\mathcal{C}(d)$, is the superalgebra generated by odd elements $c_{1}, \ldots, c_{d}$ subject to the relations $c_{i}^{2}=1$ for $i=1, \ldots, d$ and $c_{i} c_{j}=-c_{j} c_{i}$ for all $i \neq j$. There is an isomorphism

$$
\mathcal{C}\left(d_{1}+d_{2}\right) \simeq \mathcal{C}\left(d_{1}\right) \otimes \mathcal{C}\left(d_{2}\right)
$$

defined by mapping $c_{i} \mapsto c_{i} \otimes 1$ and $c_{d_{1}+j} \mapsto 1 \otimes c_{j}$, for $1 \leq i \leq d_{1}$ and $1 \leq j \leq d_{2}$. Hence, we have

$$
\mathcal{C}(d) \simeq \mathcal{C}(1) \otimes \cdots \otimes \mathcal{C}(1) \quad(d \text { copies }) .
$$

2.6. Categories enriched over $\operatorname{svec}_{\mathbb{k}}$. We say a category $\mathcal{V}$ is an $\operatorname{svec}_{\mathbb{k}}$-enriched category if the hom-sets $\operatorname{hom}_{\mathcal{V}}(V, W)(V, W \in \mathcal{V})$ are finite dimensional $\mathbb{k}$-superspaces while composition is bilinear and even. For example, if $U, V, W \in \mathcal{V}$, then composition induces an even linear map:

$$
\operatorname{hom}_{\mathcal{V}}(V, W) \otimes \operatorname{hom}_{\mathcal{V}}(U, V) \rightarrow \operatorname{hom}_{\mathcal{V}}(U, W) .
$$

We will write

$$
V \cong W
$$

if $V, W$ are isomorphic in $\mathcal{V}$. If there is an even isomorphism $\varphi: V \cong W$ (i.e., $\left.\varphi \in \operatorname{hom}_{\mathcal{V}}(V, W)_{0}\right)$, we use the notation

$$
V \simeq W
$$

Let $\mathcal{V}_{\text {ev }}$ denote the subcategory of $\mathcal{V}$ consisting of the same objects but only even homomorphisms.

For a superalgebra $\mathcal{A}$, the categories $\mathcal{A}^{\mathrm{smod}}$ and $\operatorname{smod}_{\mathcal{A}}$ are naturally $\operatorname{svec}_{\mathbb{k}^{-}}$ enriched categories. Furthermore, the subcategories $\left(\mathcal{A}^{\mathrm{smod}}\right)_{\mathrm{ev}}$ and $\left(\operatorname{smod}_{\mathcal{A}}\right)_{\mathrm{ev}}$ are abelian categories in the usual sense. This allows us to make use of the basic notions of homological algebra by restricting our attention to only even morphisms. For

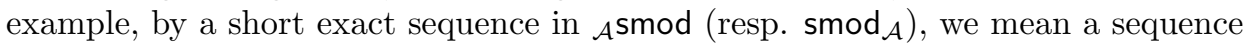

$$
0 \rightarrow V_{1} \rightarrow V_{2} \rightarrow V_{3} \rightarrow 0,
$$

with all the maps being even. All functors between the $\mathrm{svec}_{\mathbb{k}_{\mathbb{k}}}$-enriched categories which we consider will send even morphisms to even morphisms. So they will give rise to the corresponding functors between the underlying even subcategories. 
Suppose $\mathcal{V}, \mathcal{V}^{\prime}$ are $\operatorname{svec}_{\mathbb{k}}$-enriched categories. An even functor $F: \mathcal{V} \rightarrow \mathcal{V}^{\prime}$ is called $\mathbb{k}$-linear (or $\operatorname{svec}_{\mathbb{k}}$-enriched) if the map $F_{V, W}: \operatorname{hom}_{\mathcal{V}}(V, W) \rightarrow \operatorname{hom}_{\mathcal{V}^{\prime}}(F V, F W)$ is $\mathbb{k}$-linear for any pair of objects $V, W \in \mathcal{V}$. Suppose that $F, G: \mathcal{V} \rightarrow \mathcal{V}^{\prime}$ are a pair of $\mathbb{k}$-linear functors. Then an even (resp. odd) $\operatorname{svec}_{\mathbb{k}}$-enriched natural transformation $\eta: F \rightarrow G$ consists of a collection of even (resp. odd) linear maps

$$
\eta(V) \in \operatorname{hom}_{\mathcal{V}^{\prime}}(F V, G V) \quad\left({ }^{\forall} V \in \mathcal{V}\right)
$$

such that for a given $\varphi \in \operatorname{hom}_{\mathcal{V}}(V, W)$ we have

$$
G(\varphi) \circ \eta(V)=(-1)^{|\varphi||\eta(V)|} \eta(W) \circ F(\varphi) .
$$

In general, an $\mathrm{svec}_{\mathbb{k}^{-}}$-enriched natural transformation $\eta: F \rightarrow G$ is defined to be a collection of linear maps, $\eta(V)=\eta_{0}(V) \oplus \eta_{1}(V)(V \in \mathcal{V})$, such that

$$
\eta_{i}(V) \in \operatorname{hom}_{\mathcal{V}^{\prime}}(F V, G V)_{i} \quad\left(i \in \mathbb{Z}_{2}\right)
$$

and $\eta_{0}$ (resp. $\left.\eta_{1}\right): F \rightarrow G$ is an even (resp. odd) svec $_{\mathbb{k}}$-enriched natural transformation.

Now if $\mathcal{V}$ is an $\mathrm{svec}_{\mathbb{k}^{-}}$-enriched category, let $\mathcal{V}^{-}$denote the category with the same objects and morphisms as $\mathcal{V}$ but with modified composition law: $\varphi \circ \varphi^{\prime}=$ $(-1)^{|\varphi|\left|\varphi^{\prime}\right|} \varphi \varphi^{\prime}$, where $\varphi \varphi^{\prime}$ denotes composition in $\mathcal{V}$.

Example 2.2. If $f \in \operatorname{Hom}(M, N)$ for some $M, N \in \operatorname{svec}_{\mathbb{k}}$, we let $f^{-}: M \rightarrow N$ be the linear operator defined by

$$
f^{-}(v)=(-1)^{|f||v|} f(v)
$$

It can be checked that mapping $M \mapsto M$ and $f \mapsto f^{-}$for all $M, N \in \mathrm{svec}_{\mathbb{k}}$, $f \in \operatorname{Hom}(M, N)$ gives an equivalence

$$
\left(\text { svec }_{\mathbb{k}}\right)^{-} \stackrel{\sim}{\longrightarrow} \text { svec }_{\mathbb{k}} \text {. }
$$

Given a superaglebra $\mathcal{A}$, also define a new superalgebra $\mathcal{A}^{-}$, with the same elements as $\mathcal{A}$ and modified multiplication law $a \cdot b=(-1)^{|a||b|} a b$. Notice that for any $V \in \mathcal{V}$, the superspace end $\mathcal{V}(V)=\operatorname{hom}_{\mathcal{V}}(V, V)$ is a superalgebra and

$$
\operatorname{end}_{\mathcal{V}^{-}}(V)=\operatorname{end}_{\mathcal{V}}(V)^{-} \text {. }
$$

2.7. Schur's lemma. Let $\mathcal{A}$ be a superalgebra. A subsupermodule of a left (resp. right) $\mathcal{A}$-supermodule is a left (resp. right) $\mathcal{A}$-submodule, in the usual sense, which is also a subsuperspace. A left (resp. right) $\mathcal{A}$-supermodule is irreducible if it is nonzero and has no nonzero proper subsupermodules.

It is possible that an irreducible $\mathcal{A}$-supermodule may become reducible when considered as an $\overline{\mathcal{A}}$-module. We say that an irreducible left $\mathcal{A}$-supermodule $V$ is of type $M$ if the left $\overline{\mathcal{A}}$-module $\bar{V}$ is irreducible, and otherwise we say that $V$ is of type Q. We have the following criterion.

Lemma 2.3 (Schur's lemma). Suppose $\mathcal{A}$ is a superalgebra, and let $V$ be a finite dimensional irreducible left $\mathcal{A}$-supermodule. Then

$$
\operatorname{dim} \overline{\operatorname{End}_{\mathcal{A}}(V)}= \begin{cases}1 & \text { if } V \text { is of type } M, \\ 2 & \text { if } V \text { is of type } Q .\end{cases}
$$

Example 2.4. The superspace $\mathbb{k}^{m \mid n}$ is naturally an irreducible left $\mathcal{M}_{m, n}$-supermodule of type M. On the other hand, the superspace $V=\mathbb{k}^{n \mid n}$ is naturally an irreducible left $\mathcal{Q}_{n}$-supermodule. Since $\operatorname{dim} \overline{\operatorname{End}_{\mathcal{Q}_{n}}(V)}>1$, it follows that $V$ is of type $\mathbf{Q}$. This explains the given names for the types. 
2.8. Wedderburn's theorem. If $V, W \in_{\mathcal{A}} \operatorname{smod}\left(\operatorname{resp} . \operatorname{smod}_{\mathcal{A}}\right)$, we let $\operatorname{Hom}_{\mathcal{A}}(V, W)$ denote the set of $\mathcal{A}$-homomorphisms from $V$ to $W$. Also let $\operatorname{End}_{\mathcal{A}}(V)$ denote the superalgebra of all $\mathcal{A}$-supermodule endomorphisms of $V$. Given a finite dimensional superalgebra $\mathcal{A}$ and some $V \in{ }_{\mathcal{A}} \operatorname{smod}\left(\operatorname{resp}\right.$. $\left.\operatorname{smod}_{\mathcal{A}}\right)$, we have a natural isomorphism

$$
\operatorname{Hom}_{\mathcal{A}}(\mathcal{A}, V) \simeq V
$$

of vector superspaces.

We say that a left (resp. right) $\mathcal{A}$-supermodule is completely reducible if it can be decomposed as a direct sum of irreducible subsupermodules. Call $\mathcal{A}$ simple if $\mathcal{A}$ has no nontrivial superideals, and a semisimple superalgebra if $\mathcal{A}$ is completely reducible viewed as a left $\mathcal{A}$-supermodule. Equivalently, $\mathcal{A}$ is semisimple if every left $\mathcal{A}$-supermodule is completely reducible. We have:

Theorem 2.5. Let $\mathcal{A}$ be a finite dimensional superalgebra. The following are equivalent:

(i) $\mathcal{A}$ is semisimple;

(ii) every left (resp. right) $\mathcal{A}$-supermodule is completely reducible;

(iii) $\mathcal{A}$ is a direct product of finitely many simple superalgebras.

Example 2.6. The Clifford superalgebra $\mathcal{C}(1)$ may be realized as the superalgebra of $2 \times 2$ matrices of the form $\left\{\left(\begin{array}{ll}a & b \\ b & a\end{array}\right) \mid a, b \in \mathbb{k}\right\}$. The generator $c_{1}$ of $\mathcal{C}(1)$ corresponds to the matrix $J_{1}=\left(\begin{array}{ll}0 & 1 \\ 1 & 0\end{array}\right)$. One may check that $\mathcal{C}(1)$ is a simple superalgebra with a unique right (resp. left) irreducible supermodule up to isomorphism. In fact, $\mathcal{C}(1)$ is an irreducible supermodule over itself with respect to right (resp. left) multiplication, and we denote this supermodule by $\mathcal{U}_{r}(1)$ (resp. $\mathcal{U}_{l}(1)$ ). In the sequel, we usually write $\mathcal{U}(1)=\mathcal{U}_{r}(1)$.

Suppose that $V \in \mathcal{C}(1)_{\text {smod, }} V^{\prime} \in \operatorname{smod}_{\mathcal{C}(1)}$. By Theorem 2.5, there exist decompositions $V \simeq \mathcal{U}_{l}(1)^{\oplus n}$ and $V^{\prime} \simeq \mathcal{U}(1)^{\oplus n^{\prime}}$, for some $n, n^{\prime} \in \mathbb{Z}_{\geq 0}$. Hence, we have $\operatorname{sdim}(V)=(n, n)$ and $\operatorname{sdim}\left(V^{\prime}\right)=\left(n^{\prime}, n^{\prime}\right)$, and there exists a basis of $V\left(\right.$ resp. $\left.V^{\prime}\right)$ such that $c_{1} \in \mathcal{C}(1)$ acts on $V\left(\right.$ resp. $\left.V^{\prime}\right)$ via multiplication by the matrix

$$
\left(\begin{array}{cc}
0 & I_{N} \\
I_{N} & 0
\end{array}\right)
$$

where $I_{N}$ is the $N \times N$ unit matrix for $N=n, n^{\prime}$ respectively.

Now let $V, W \in \mathcal{C}_{(1)} \operatorname{smod}\left(\operatorname{resp} . \operatorname{smod}_{\mathcal{C}(1)}\right)$. As mentioned above, we may assume that $\operatorname{sdim}(V)=(m, m)($ resp. $\operatorname{sdim}(W)=(n, n))$ for some $m, n \in \mathbb{Z}_{\geq 0}$. By equation (10), we may choose respective bases of $V$ and $W$ such that $\operatorname{Hom}_{\mathcal{C}(1)}(V, W)$ consists of all matrices of the form

$$
\left(\begin{array}{cc}
A & B \\
-B & A
\end{array}\right) \quad\left(\operatorname{resp} .\left(\begin{array}{cc}
A & B \\
B & A
\end{array}\right)\right)
$$

where $A, B$ are $n \times m$ matrices in $\mathbb{k}$, and $A=0$ (resp. $B=0)$ for odd (resp. even) homomorphisms.

Remark 2.7. Notice that $\overline{\mathcal{C}(1)}$ is commutative as an ordinary algebra even though $\mathcal{C}(1)$ is not a commutative superalgebra. Hence, the objects of $\mathcal{C}(1)$ smod can be identified with the objects of $\operatorname{smod}_{\mathcal{C}(1)}$. It can be checked using (11) that we have an equivalence

$$
\left(\mathcal{C}(1) \mathrm{smod}^{-} \stackrel{\sim}{\longrightarrow} \operatorname{smod}_{\mathcal{C}(1)},\right.
$$


given by mapping $V \mapsto V$ and $\varphi \mapsto \varphi^{-}$for all $V, W \in(\mathcal{C}(1) \text { smod })^{-}$and $\varphi \in$ $\operatorname{Hom}_{\mathcal{C}(1)}(V, W)$.

Remark 2.8. Suppose that $V \in \mathcal{C}(1) \operatorname{smod}$ and $\operatorname{sdim}(V)=(n, n)$. Then it is clear from (11) that we have a superalgebra isomorphism $\mathcal{Q}_{n} \cong \operatorname{End}_{\mathcal{C}(1)}(V)$. Now suppose that there is a $\sqrt{-1} \in \mathbb{k}$. If $V^{\prime} \in \operatorname{smod}_{\mathcal{C}(1)}$ and again $\operatorname{sdim}\left(V^{\prime}\right)=(n, n)$, then it is not difficult to check that we also have an isomorphism $\mathcal{Q}_{n} \cong \operatorname{End}_{\mathcal{C}(1)}\left(V^{\prime}\right)$ of superalgebras.

2.9. Wreath products. Suppose $\mathcal{A}$ is an associative superalgebra, and let $d \in$ $\mathbb{Z}_{\geq 1}$. Notice that the right action of $\sigma \in \mathfrak{S}_{d}$ on the tensor power $\mathcal{A}^{\otimes d}$ is in fact a superalgebra automorphism. Denote by $\mathcal{A} \prec \mathfrak{S}_{d}$ the vector superspace

$$
\mathcal{A}<\mathfrak{S}_{d}=\mathbb{k} \mathfrak{S}_{d} \otimes \mathcal{A}^{\otimes d}
$$

(where the group algebra $\mathbb{k} \mathfrak{S}_{d}$ is viewed as superspace concentrated in degree zero). We then consider $\mathcal{A} \prec \mathfrak{S}_{d}$ as a superalgebra with multiplication defined by the rule

$$
(\sigma \otimes a)\left(\sigma^{\prime} \otimes b\right)=\sigma \sigma^{\prime} \otimes\left(a \cdot \sigma^{\prime}\right) b
$$

for $\sigma, \sigma^{\prime} \in \mathfrak{S}_{d}, a, b \in \mathcal{A}$. In what follows, we will identify $\mathcal{A}^{\otimes d}\left(\right.$ resp. $\mathbb{k} \mathfrak{S}_{d}$ ) with the subsuperalgebra $1 \otimes \mathcal{A}^{\otimes d}$ (resp. $\left.\mathbb{k}_{d} \otimes 1\right)$ of $\mathcal{A}$ 々 $\mathfrak{S}_{d}$.

Example 2.9 (Sergeev superalgebra). If $\mathcal{A}=\mathbb{k}$, then $\mathbb{k} \iota \mathfrak{S}_{d}=\mathbb{k} \mathfrak{S}_{d}$, the group algebra of $\mathfrak{S}_{d}$. On the other hand, if we identity $C(d)$ with $\mathcal{C}(1)^{\otimes d}$ via the isomorphism (6), then $\mathcal{C}(1)<\mathfrak{S}_{d}=\mathcal{W}(d)$, the Sergeev superalgebra (cf. [BrK1]).

2.10. Tensor products of supermodules. Given left supermodules $V$ and $W$ over arbitrary superalgebras $\mathcal{A}$ and $\mathcal{B}$ respectively, the tensor product $V \otimes W$ is a left $\mathcal{A} \otimes \mathcal{B}$-supermodule with action defined by $(a \otimes b) .(v \otimes w)=(-1)^{|b||v|} a . v \otimes b . w$, for all homogeneous $a \in \mathcal{A}, b \in \mathcal{B}, v \in V, w \in W$. (Analogously, if $V$ and $W$ are right supermodules, the action of $\mathcal{A} \otimes \mathcal{B}$ on $V \otimes W$ is given by $(v \otimes w) \cdot(a \otimes b)=$ $(-1)^{|w||a|} v . a \otimes w . b$, for all homogeneous $a \in \mathcal{A}, b \in \mathcal{B}, v \in V, w \in W$.) If $\varphi: V \rightarrow V^{\prime}$ (resp. $\varphi^{\prime}: W \rightarrow W^{\prime}$ ) is a homogeneous homomorphism of left $\mathcal{A}$ - (resp. $\mathcal{B}$-) supermodules, then $\varphi \otimes \varphi^{\prime}: V \otimes W \rightarrow V^{\prime} \otimes W^{\prime}$ is a homomorphism of left $\mathcal{A} \otimes \mathcal{B}$ supermodules, where

$$
\varphi \otimes \varphi^{\prime}(v \otimes w)=(-1)^{\left|\varphi^{\prime}\right||v|} \varphi v \otimes \varphi^{\prime} w .
$$

(The previous statement holds also for right supermodules. For example, the outer tensor product $\varphi \otimes \varphi^{\prime}$ of right supermodule homomorphisms, $\varphi: V \rightarrow W$ and $\varphi^{\prime}: V^{\prime} \rightarrow W^{\prime}$, is given by the same formula (13).)

As a particular example, if $M, M^{\prime}, N, N^{\prime} \in \mathrm{svec}_{\mathbb{k}}$, then (13) gives a natural isomorphism

$$
\operatorname{Hom}_{\mathbb{k}}(M, N) \otimes \operatorname{Hom}_{\mathbb{k}}\left(M^{\prime}, N^{\prime}\right) \simeq \operatorname{Hom}_{\mathbb{k}}\left(M \otimes M^{\prime}, N \otimes N^{\prime}\right),
$$

which sends $f \otimes f^{\prime} \mapsto f \otimes f^{\prime}$. More generally, we have the following.

Lemma 2.10. Suppose $\mathcal{B}$ is a simple finite dimensional superalgebra. If $V, W \in$ $\operatorname{smod}_{\mathcal{B}}$, then there is a canonical isomorphism

$$
\operatorname{Hom}_{\mathcal{B}}(V, W)^{\otimes d} \simeq \operatorname{Hom}_{\mathcal{B} \otimes d}\left(V^{\otimes d}, W^{\otimes d}\right),
$$

which maps $f_{1} \otimes \cdots \otimes f_{d}$ onto $f_{1} \otimes \cdots \otimes f_{d}$.

Proof. It suffices to consider $d=2$. The map $f \otimes g \mapsto f \otimes g$ is clearly injective. To check that it is surjective we may use Lemma 2.3 together with Theorem 2.5 and [K, Lemma 12.2.13]. 


\section{StRict POlynomial FUnCtors of types I AND II}

We now introduce the categories Pol $_{d, \mathbb{k}}^{(\mathrm{I})}$ and Pol ${ }_{d, \mathbb{k}}^{(\mathrm{II})}$ consisting of homogeneous strict polynomial functors. Such polynomial functors are realized as $\mathbb{k}$-linear functors between an appropriate pair of $\mathrm{svec}_{\mathbb{k}_{\mathrm{k}}}$-enriched categories.

3.1. Categories of divided powers. Suppose that $\mathcal{B}$ is a simple finite dimensional superalgebra, and let $\mathcal{V}=\operatorname{smod}_{\mathcal{B}}$. For each $d \in \mathbb{Z}_{\geq 0}$, we define a new category $\Gamma^{d} \mathcal{V}$. The objects of $\Gamma^{d} \mathcal{V}$ are the same as those of $\mathcal{V}$, i.e., finite dimensional right $\mathcal{B}$-supermodules. Given $V, W \in \operatorname{smod}_{\mathcal{B}}$, set

$$
\operatorname{hom}_{\Gamma^{d} \mathcal{V}}(V, W):=\Gamma^{d} \operatorname{Hom}_{\mathcal{B}}(V, W) .
$$

In order to define the composition law, we make use of the following lemma.

Lemma 3.1. Suppose $V \in \operatorname{smod}_{\mathcal{B}}$. Then $V^{\otimes d} \in \operatorname{smod}_{\mathcal{B} \backslash \mathfrak{S}_{d}}$, where $\mathcal{B}\left\{\mathfrak{S}_{d}\right.$ is the wreath product defined above. If $V, W \in \operatorname{smod}_{\mathcal{B}}$, then we further have a natural isomorphism

$$
\operatorname{Hom}_{\mathcal{B} \imath \mathfrak{S}_{d}}\left(V^{\otimes d}, W^{\otimes d}\right) \simeq \Gamma^{d} \operatorname{Hom}_{\mathcal{B}}(V, W) .
$$

Proof. By Lemma 2.10, $V^{\otimes d} \in \operatorname{smod}_{\mathcal{B} \otimes d}$. One may check that for any $\sigma \in \mathfrak{S}_{d}$, we have

$$
(v \cdot a) . \sigma=(v \cdot \sigma) \cdot(a \cdot \sigma) \quad\left(v \in V^{\otimes d}, a \in \mathcal{B}^{\otimes d}\right),
$$

where $\mathfrak{S}_{d}$ acts on $\mathcal{B}^{\otimes d}$ on the right as in the definition of $\mathcal{B} \backslash \mathfrak{S}_{d}$. Now given a homomorphism $\varphi \in \operatorname{Hom}_{\mathcal{B} \otimes d}\left(V^{\otimes d}, W^{\otimes d}\right)$, it follows from (17) that $\varphi^{\sigma} \in \operatorname{Hom}_{\mathcal{B} \otimes d}\left(V^{\otimes d}\right.$, $\left.W^{\otimes d}\right)$, where $\varphi^{\sigma}: V^{\otimes d} \rightarrow W^{\otimes d}$ is the linear map defined by $\varphi^{\sigma}(v)=\left(\varphi\left(v \cdot \sigma^{-1}\right)\right) . \sigma$ for any $v \in V^{\otimes d}$. One may then check that

$$
\operatorname{Hom}_{\mathcal{B} \otimes d}\left(V^{\otimes d}, W^{\otimes d}\right)^{\mathfrak{S}_{d}}=\operatorname{Hom}_{\mathcal{B}_{\imath} \mathfrak{S}_{d}}\left(V^{\otimes d}, W^{\otimes d}\right) .
$$

It is also not difficult to check that the isomorphism (15) is in fact an isomorphism of $\mathfrak{S}_{d}$-modules. Hence we have a canonical isomorphism

$$
\begin{aligned}
\Gamma^{d} \operatorname{Hom}_{\mathcal{B}}(V, W) & =\left(\operatorname{Hom}_{\mathcal{B}}(V, W)^{\otimes d}\right)^{\mathfrak{S}_{d}} \\
& \simeq \operatorname{Hom}_{\mathcal{B} \otimes d}\left(V^{\otimes d}, W^{\otimes d}\right)^{\mathfrak{S}_{d}}=\operatorname{Hom}_{\mathcal{B} \backslash \mathfrak{S}_{d}}\left(V^{\otimes d}, W^{\otimes d}\right) .
\end{aligned}
$$

Using the isomorphism in the previous lemma for any $V, W \in \mathcal{V}=\operatorname{smod}_{\mathcal{B}}$, composition in $\operatorname{smod}_{\mathcal{B} \mathfrak{S}_{d}}$ induces a composition law in $\Gamma^{d} \mathcal{V}$. As primary examples, we have the categories $\Gamma_{\mathrm{M}}^{d}=\Gamma^{d}$ svec $_{\mathbb{k}}$ and $\Gamma_{\mathrm{Q}}^{d}=\Gamma^{d} \operatorname{smod}_{\mathcal{C}(1)}$.

3.2. Schur superalgebras. Let $M=\mathbb{k}^{m \mid n}$. Then we have a superalgebra isomorphism

$$
\operatorname{end}_{\Gamma_{\mathrm{M}}^{d}}(M)=\operatorname{End}_{\mathbb{k} \mathfrak{S}_{d}}\left(M^{\otimes d}\right) \cong \mathcal{S}(m \mid n, d),
$$

where $\mathcal{S}(m \mid n, d)$ is the Schur superalgebra defined in [D].

Let $V=\mathcal{U}(1)^{\oplus n} \in \Gamma_{\mathrm{Q}}^{d}$. Then we have another isomorphism of superalgebras

$$
\operatorname{end}_{\Gamma_{Q}^{d}}(V)=\operatorname{End}_{\mathcal{W}(d)}\left(V^{\otimes d}\right) \cong \mathcal{Q}(n, d),
$$

where $\mathcal{Q}(n, d)$ is the Schur superalgebra defined in BrK1. 
3.3. Strict polynomial functors. Notice that $\Gamma_{\mathrm{M}}^{d}$ and $\Gamma_{\mathrm{Q}}^{d}$ are both $\mathrm{svec}_{\mathbb{R}}$-enriched categories. Let $\mathrm{Pol}_{d}^{(\mathrm{I})}=\operatorname{Fct}_{\mathbb{k}}\left(\Gamma_{\mathrm{M}}^{d}, \mathrm{svec}_{\mathbb{k}}\right)$, the category of (even) $\mathbb{k}$-linear functors from $\Gamma_{\mathrm{M}}^{d}$ to $\mathrm{svec}_{\mathbb{k}}$. Similarly, let $\mathrm{Pol}_{d}^{(\mathrm{II})}=\operatorname{Fct}_{\mathbb{k}}\left(\Gamma_{\mathrm{Q}}^{d}, \operatorname{svec}_{\mathbb{k}}\right)$. In both cases, morphisms are $\mathrm{svec}_{\mathbb{k}_{\mathrm{k}}}$-enriched natural transformations (see Section 2.6), and objects of either category are called (homogeneous) strict polynomial functors. In the particular case $d=0$, all objects of $\mathrm{Pol}_{0}^{(\mathrm{I})}$ and $\mathrm{Pol}_{0}^{(\mathrm{II})}$ are equivalent to constant functors.

Given $S, T \in \mathrm{Pol}_{d}^{(\mathrm{I})}$ (resp. $\mathrm{Pol}_{d}^{(\mathrm{II})}$ ), the set of all natural transformations $\eta$ : $S \rightarrow T$ is naturally a vector superspace. In this way, we see that $\mathrm{Pol}_{d}^{(\mathrm{I})}$ and $\mathrm{Pol}_{d}^{(\mathrm{II})}$ are $\mathrm{svec}_{\mathbb{k}_{\mathrm{k}}}$-enriched categories. The even subcategories $\left(\mathrm{Pol}_{d}^{(\mathrm{I})}\right)_{\mathrm{ev}},\left(\mathrm{Pol}_{d}^{(\mathrm{II})}\right)_{\mathrm{ev}}$ both inherit the structure of an abelian category, since kernels, cokernels, products and coproducts can be computed in the target category $\left(\mathrm{svec}_{\mathbb{k}}\right)_{\mathrm{ev}}$.

Examples 3.2. We have the following examples of strict polynomial functors belonging to $\mathrm{Pol}_{d}^{(\mathrm{I})}$ for some $d \geq 1$.

(i) The identity functor, Id $: \operatorname{svec}_{\mathbb{k}} \rightarrow \mathrm{svec}_{\mathbb{k}}$, belongs to Pol ${ }_{1}^{(\mathrm{I})}$. Another object of $\mathrm{Pol}_{1}^{(\mathrm{I})}$ is the parity change functor $\Pi: \mathrm{svec}_{\mathbb{k}} \rightarrow \mathrm{svec}_{\mathbb{k}}$, introduced in the previous section.

(ii) The functor $\otimes^{d} \in \mathrm{Pol}_{d}^{(\mathrm{I})}$ sends an object $M \in \Gamma_{\mathrm{M}}^{d}$ to $M^{\otimes d}$ and a morphism $f \in \operatorname{hom}_{\Gamma_{M}^{d}}(M, N)$ to the same underlying map regarded as an element of $\operatorname{Hom}_{\mathbb{k}}\left(M^{\otimes d}, N^{\otimes d}\right)$.

(iii) Given $M \in \operatorname{svec}_{\mathbb{k}}$, let $\Gamma^{d, M}=\operatorname{hom}_{\Gamma_{M}^{d}}(M,-)$, a representable functor in Pol $_{d}^{(\mathrm{I})}$. In particular, if $M=\mathbb{k}^{m \mid n}$ we write $\Gamma^{d, m \mid n}=\Gamma^{d, M}$.

Notice that for any $M \in \operatorname{svec}_{\mathbb{k}}$, we have a canonical isomorphism

$$
\Gamma^{d} M \simeq \Gamma^{d, 1 \mid 0}(M),
$$

since $\operatorname{hom}_{\Gamma_{M}^{d}}(\mathbb{k}, M)=\Gamma^{d} \operatorname{Hom}_{\mathbb{k}}(\mathbb{k}, M) \simeq \Gamma^{d} M$.

Let us identify $\operatorname{smod}_{\mathcal{C}(1)}$ as a subcategory of $\operatorname{svec}_{\mathbb{k}}$. Since we may view $\mathbb{k} \mathfrak{S}_{d}$ as a subsuperalgebra of $\mathcal{W}(d)$, there is a restriction functor from $\operatorname{smod}_{\mathcal{W}(d)}$ to $\operatorname{smod}_{\mathbb{k} \mathfrak{S}_{d}}$. This in turn yields an even $\mathbb{k}$-linear functor, Res : $\Gamma_{Q}^{d} \rightarrow \Gamma_{\mathrm{M}}^{d}$, which acts as the identity on objects and by restriction on morphisms. Hence, composition yields a functor

$$
-\circ \operatorname{Res}: \mathrm{Pol}_{d}^{(\mathrm{I})} \rightarrow \mathrm{Pol}_{d}^{(\mathrm{II})}
$$

Examples 3.3. The following are examples of objects in $\mathrm{Pol}_{d}^{(\mathrm{II})}$, for some $d \geq 1$.

(i) We use the same notation, Id $=\mathrm{Id} \circ \operatorname{Res}: \operatorname{smod}_{\mathcal{C}(1)} \rightarrow \mathrm{svec}_{\mathbb{k}}$, to denote the restriction of the identity functor. Clearly $\mathrm{Id} \in \mathrm{Pol}_{1}^{(\mathrm{II})}$. Also, note that we have an even isomorphism

$$
\Pi \circ \operatorname{Res} \simeq \mathrm{Id}
$$

in $\mathrm{Pol}_{1}^{(\mathrm{II})}$.

(ii) The functor $\otimes^{d}=\otimes^{d} \circ \operatorname{Res} \in \mathrm{Pol}_{d}^{(\mathrm{II})}$ sends an object $V \in \Gamma_{\mathrm{Q}}^{d}$ to $V^{\otimes d}$ and a morphism $\varphi \in \operatorname{hom}_{\Gamma_{Q}^{d}}(V, W)$ to the same underlying map regarded as an element of $\operatorname{Hom}_{\mathbb{k}}\left(V^{\otimes d}, W^{\otimes d}\right)$.

(iii) If $V \in \Gamma_{Q}^{d}$, let $\Gamma^{d, V}=\operatorname{hom}_{\Gamma_{Q}^{d}}(V,-)$, which belongs to Pol ${ }_{d}^{(I)}$. In case $V=$ $\mathcal{U}(1)^{\oplus n}$, we write $\Gamma^{d, n}=\Gamma^{d, V}$. 
Given $V \in \operatorname{smod}_{\mathcal{C}(1)}$, notice that we have a canonical isomorphism

$$
\Gamma^{d, 1}(V) \simeq \Gamma^{d} V
$$

since

$$
\Gamma^{d} \operatorname{Hom}_{\mathcal{C}(1)}(\mathcal{U}(1), V)=\Gamma^{d} \operatorname{Hom}_{\mathcal{C}(1)}(\mathcal{C}(1), V) \simeq \Gamma^{d} V .
$$

3.4. Duality. Suppose $\tau$ is an antiautomorphism of a superalgebra $\mathcal{B}$, and let $V \in \operatorname{smod}_{\mathcal{B}}$. Then we can make the dual space $V^{\vee}$ into a right $\mathcal{B}$-supermodule by defining

$$
\langle f . a, v\rangle=\langle f, v . \tau(a)\rangle \quad\left(a \in \mathcal{B}, f \in V^{\vee}, v \in V\right) .
$$

We denote the resulting supermodule by $V^{\tau, \vee}$. If $V, W \in \operatorname{smod}_{\mathcal{B}}$ and $\varphi \in \operatorname{Hom}_{\mathcal{B}}(V, W)$, then let $\varphi^{\vee}: W^{\tau, \vee} \rightarrow V^{\tau, \vee}$ be defined by

$$
\left\langle\varphi^{\vee}(f), v\right\rangle=(-1)^{|\varphi||f|}\langle f, \varphi(v)\rangle
$$

for all $f \in V^{\vee}, v \in V$. Then $\varphi^{\vee} \in \operatorname{Hom}_{\mathcal{B}}\left(W^{\tau, \vee}, V^{\tau, \vee}\right)$, and furthermore we have a natural isomorphism

$$
\operatorname{Hom}_{\mathcal{B}}(V, W) \simeq \operatorname{Hom}_{\mathcal{B}}\left(W^{\tau, \vee}, V^{\tau, \vee}\right) .
$$

Given any $\mathrm{svec}_{\mathbb{k}^{-}}$-enriched category $\mathcal{V}$, let us write $\mathcal{V}^{\mathrm{op},-}=\left(\mathcal{V}^{-}\right)^{\text {op }}$ to denote the opposite category of $\mathcal{V}^{-}$. Now let $\mathcal{V}=\operatorname{smod}_{\mathcal{B}}$. Then (20) gives an equivalence of categories

$$
()^{\tau, \vee}:\left(\operatorname{smod}_{\mathcal{B}}\right)^{\mathrm{op},-} \stackrel{\sim}{\longrightarrow} \operatorname{smod}_{\mathcal{B}} .
$$

An antiautomorphism $\tau$ of $\mathcal{B}$ induces an antiautomorphism $\tau_{2}$ of $\mathcal{B} \otimes \mathcal{B}$ by setting $\tau_{2}(a \otimes b)=(-1)^{|a||b|} \tau(a) \otimes \tau(b)$. In general, this gives an antiautomorphism $\tau_{d}$ of $\mathcal{B}^{\otimes d}$ for all $d \geq 1$. If $V, W \in \operatorname{smod}_{\mathcal{B}}$, we have a canonical isomorphism of $\mathcal{B} \otimes \mathcal{B}$ supermodules

$$
V^{\tau, \vee} \otimes W^{\tau, \vee} \simeq(V \otimes W)^{\tau_{2}, \vee}
$$

given by

$$
\langle f \otimes g, v \otimes w\rangle=(-1)^{|g||v|}\langle f, v\rangle\langle g, w\rangle
$$

for all $f \in V^{\tau, \vee}, g \in W^{\tau, \vee}, v \in V, w \in W$.

Suppose now that $\mathcal{B}$ is a simple finite dimensional superalgebra. Let us fix generators $s_{i}=(i i+1) \in \mathfrak{S}_{d}$ for $i=1, \ldots, d-1$. Then $\tau_{d}$ extends uniquely to an antiautomorphism of $\mathcal{B}_{2} \mathfrak{S}_{d}$, also denoted $\tau_{d}$, such that $\tau_{d}\left(s_{i}\right)=s_{i}$ for $i=1, \ldots, d-1$. So that the equivalence (21) with respect to $\mathcal{B} \imath \mathfrak{S}_{d}$ and $\tau_{d}$ induces a correspoding equivalence

$$
()^{\tau, \vee}:\left(\Gamma^{d} \mathcal{V}\right)^{\mathrm{op},-} \stackrel{\sim}{\longrightarrow} \Gamma^{d} \mathcal{V}
$$

for $\mathcal{V}=\operatorname{smod}_{\mathcal{B}}$.

Example 3.4. If $\mathcal{B}=\mathbb{k}$ or $\mathcal{C}(1)$, then setting $\tau(a)=a\left({ }^{\forall} a \in \mathcal{B}\right)$ defines an antiautomorphism of $\mathcal{B}$. Hence we have equivalences

$$
()^{\vee}:\left(\Gamma_{\mathrm{M}}^{d}\right)^{\mathrm{op},-} \stackrel{\sim}{\longrightarrow} \Gamma_{\mathrm{M}}^{d} \quad \text { and } \quad()^{\vee}:\left(\Gamma_{\mathrm{Q}}^{d}\right)^{\mathrm{op},-} \stackrel{\sim}{\longrightarrow} \Gamma_{\mathrm{Q}}^{d} .
$$

Again let $\mathcal{V}=\operatorname{smod}_{\mathcal{B}}$ for a finite dimensional simple superalgebra $\mathcal{B}$. Suppose $T \in \operatorname{Fct}_{\mathbb{k}}\left(\Gamma^{d} \mathcal{V}, \operatorname{svec}_{\mathbb{k}}\right)$. Then define the Kuhn dual

$$
T^{\#} \in \operatorname{Fct}_{\mathbb{k}}\left(\left(\Gamma^{d} \mathcal{V}\right)^{\mathrm{op},-},\left(\operatorname{svec}_{\mathbb{k}}\right)^{\mathrm{op},-}\right)=\operatorname{Fct}_{\mathbb{k}}\left(\Gamma^{d} \mathcal{V}, \operatorname{svec}_{\mathbb{k}}\right)
$$


by setting $T^{\#}(V)=T\left(V^{\tau, \vee}\right)^{\vee}$ for all $V \in \mathcal{V}$. If $\mathcal{B}=\mathbb{k}$ or $\mathcal{C}(1)$, this gives an equivalence

$$
()^{\#}:\left(\mathrm{Pol}_{d}^{(\dagger)}\right)^{\mathrm{op},-} \stackrel{\sim}{\longrightarrow} \mathrm{Pol}_{d}^{(\dagger)},
$$

for $\dagger=\mathrm{I}$ or II, respectively.

As an example, for $V \in \Gamma_{\mathrm{M}}^{d}\left(\operatorname{resp} . \Gamma_{\mathrm{Q}}^{d}\right)$, we define $S^{d, V}:=\left(\Gamma^{d, V}\right)^{\#}$. In particular, let us write $S^{d, m \mid n}=S^{d, \mathbb{k}^{m \mid n}}$ and $S^{d, n}=S^{d, \mathcal{U}(1)^{\oplus n}}$. It then follows from equation (3) that we have canonical isomorphisms

$$
S^{d, 1 \mid 0}(M) \simeq S^{d} M, \quad S^{d, 1}(V) \simeq S^{d} V
$$

for all $M \in \operatorname{svec}_{\mathbb{k}}, V \in \operatorname{smod}_{\mathcal{C}(1)}$ respectively.

3.5. Yoneda's lemma. We have the following analogue of Yoneda's lemma in our setting.

Lemma 3.5. Suppose that $T \in \mathrm{Pol}_{d}^{(\mathrm{I})}$ and $T^{\prime} \in \mathrm{Pol}_{d}^{(\mathrm{II})}$. Then we have natural isomorphisms:

$$
\begin{aligned}
& \text { (i) } \operatorname{hom}_{\mathrm{Pol}_{d}^{(\mathrm{I})}}\left(\Gamma^{d, M}, T\right) \simeq T(M) \quad\left(M \in \operatorname{svec}_{\mathbb{k}}\right) \text {, and } \\
& \text { (ii) } \operatorname{hom}_{\mathrm{Pol}_{d}^{(\mathrm{II})}}\left(\Gamma^{d, V}, T^{\prime}\right) \simeq T^{\prime}(V) \quad\left(V \in \operatorname{smod}_{\mathcal{C}(1)}\right) .
\end{aligned}
$$

It follows that $\Gamma^{d, M}, \Gamma^{d, V}$ are projective objects of $\left(\mathrm{Pol}_{d}^{(\mathrm{I})}\right)_{\mathrm{ev}},\left(\mathrm{Pol}_{d}^{(\mathrm{II})}\right)_{\mathrm{ev}}$ respectively. On the other hand, the dual objects $S^{d, M}, S^{d, V}$ are injective by Kuhn duality (23).

3.6. Tensor products. Given nonnegative integers $d$ and $e$, we have an embedding $\mathfrak{S}_{d} \times \mathfrak{S}_{e} \hookrightarrow \mathfrak{S}_{d+e}$. This induces an embedding

$$
\Gamma^{d+e} M \hookrightarrow \Gamma^{d} M \otimes \Gamma^{e} M,
$$

for any $M \in \mathrm{svec}_{\mathbb{k}}$, given by the composition of the following maps

$$
\begin{aligned}
\Gamma^{d+e} M=\left(M^{\otimes(d+e)}\right)^{\mathfrak{S}_{d+e}} & \subseteq\left(M^{\otimes d+e)}\right)^{\mathfrak{S}_{d} \times \mathfrak{S}_{e}} \\
& \simeq\left(M^{\otimes d}\right)^{\mathfrak{S}_{d}} \otimes\left(M^{\otimes e}\right)^{\mathfrak{S}_{e}}=\Gamma^{d} M \otimes \Gamma^{e} M .
\end{aligned}
$$

Now we may consider the categories $\Gamma_{M}^{d} \otimes \Gamma_{M}^{e}, \Gamma_{Q}^{d} \otimes \Gamma_{Q}^{e}$ whose objects are the same as $\operatorname{svec}_{\mathbb{k}}, \operatorname{smod}_{\mathcal{C}(1)}$ and whose morphisms are of the form

$$
\operatorname{hom}_{\Gamma_{\mathrm{M}}^{d}}(M, N) \otimes \operatorname{hom}_{\Gamma_{\mathrm{M}}^{e}}(M, N), \quad \operatorname{hom}_{\Gamma_{\mathrm{Q}}^{d}}(V, W) \otimes \operatorname{hom}_{\Gamma_{\mathrm{Q}}^{e}}(V, W)
$$

respectively for $M, N \in \operatorname{svec}_{\mathbb{k}}$ and $V, W \in \operatorname{smod}_{\mathcal{C}(1)}$. Then, one may show that (24) yields embeddings of categories

$$
\Gamma_{\mathrm{M}}^{d+e} \hookrightarrow \Gamma_{\mathrm{M}}^{d} \otimes \Gamma_{\mathrm{M}}^{e}, \quad \Gamma_{\mathrm{Q}}^{d+e} \hookrightarrow \Gamma_{\mathrm{Q}}^{d} \otimes \Gamma_{\mathrm{Q}}^{e}
$$

Now suppose $S \in \mathrm{Pol}_{d}^{(\dagger)}, T \in \mathrm{Pol}_{e}^{(\dagger)}(\dagger=\mathrm{I}, \mathrm{II})$. Let $S \otimes T \in \mathrm{Pol}_{d+e}^{(\dagger)}$ denote the functor defined by setting: for $V, W \in \operatorname{svec}_{\mathbb{k}}\left(\operatorname{resp} . \operatorname{smod}_{\mathcal{C}(1)}\right)$ and $\varphi: V \rightarrow W$ a $\mathbb{k}$-linear (resp. $\mathcal{C}(1)$-linear) map,

$$
(S \otimes T)(V):=S(V) \otimes T(V) \text { and }(S \otimes T)(\varphi):=S(\varphi) \otimes T(\varphi),
$$

respectively. Then (25) induces bifunctors:

$$
-\otimes-: \mathrm{Pol}_{d}^{(\dagger)} \times \mathrm{Pol}_{e}^{(\dagger)} \rightarrow \mathrm{Pol}_{d+e}^{(\dagger)} \quad(\dagger=\mathrm{I}, \mathrm{II}),
$$

which respectively send $S \times T \mapsto S \otimes T$. 


\section{Strict polynomial functors, Schur superalgebras and SERGEEEV DUALITY}

We show that the categories of strict polynomial functors of types I and II defined above are equivalent to categories of supermodules for the Schur superalgebras $\mathcal{S}(m \mid n, d)$ and $\mathcal{Q}(n, d)$, respectively. We also describe a functorial analogue of Sergeev duality for type II strict polynomial functors.

4.1. Equivalences of categories. Let $M \in \operatorname{svec}_{\mathbb{k}}$. If $v \in M$, we write $v^{\otimes d}=$ $v \otimes \cdots \otimes v$ ( $d$ factors). Suppose that $I=\left(d_{1}, \ldots, d_{s}\right)$ is a tuple of positive integers, and let $\mathfrak{S}_{I}$ denote the subgroup $\mathfrak{S}_{d_{1}} \times \cdots \times \mathfrak{S}_{d_{s}} \subseteq \mathfrak{S}_{|I|}$, where $|I|=\sum d_{i}$. Given distinct nonzero elements $v_{1}, \ldots, v_{s} \in M_{0}$, we define the new element

$$
\left(v_{1}, \ldots, v_{s} ; I\right)_{0}:=\sum_{\sigma \in \mathfrak{S}_{|I|} / \mathfrak{S}_{I}}\left(v_{1}^{\otimes d_{1}} \otimes \cdots \otimes v_{s}^{\otimes d_{s}}\right) . \sigma
$$

which belongs to $\left(M_{0}^{\otimes|I|}\right)^{\mathfrak{S}_{|I|}}$. Similiarly, if $v_{1}^{\prime}, \ldots, v_{t}^{\prime} \in M_{1}$, we define the (possibly zero) element

$$
\left(v_{1}^{\prime}, \ldots, v_{t}^{\prime}\right)_{1}:=\sum_{\sigma \in \mathfrak{S}_{t}}\left(v_{1}^{\prime} \otimes \cdots \otimes v_{t}^{\prime}\right) \cdot \sigma
$$

which belongs to $\left(M_{1}^{\otimes t}\right)^{\mathfrak{S}_{t}}$.

Lemma 4.1. Let $M \in \operatorname{svec}_{\mathbb{k}}$, and suppose $\left\{e_{1}^{(0)}, \ldots, e_{m}^{(0)}\right\},\left\{e_{1}^{(1)}, \ldots, e_{n}^{(1)}\right\}$ are ordered bases of $M_{0}, M_{1}$ respectively. Then $\Gamma^{d} M$ has a basis given by the set of all elements of the form

$$
\left(e_{i_{1}}^{(0)}, \ldots, e_{i_{s}}^{(0)} ; I\right)_{0} \cdot\left(e_{j_{1}}^{(1)}, \ldots, e_{j_{t}}^{(1)}\right)_{1},
$$

such that $|I|+t=d, 1 \leq i_{1}<\cdots<i_{s} \leq m$ and $1 \leq j_{1}<\cdots<j_{t} \leq n$.

Proof. It follows from (4) that we have isomorphisms of superspaces

$$
\Gamma^{d} M \simeq \bigoplus_{k+l=d} D^{k}\left(\overline{M_{0}}\right) \otimes \Lambda^{l}\left(\overline{M_{1}}\right) \simeq \bigoplus_{k+l=d} \Gamma^{k} M_{0} \cdot \Gamma^{l} M_{1}
$$

for each $d \geq 0$.

One may check by comparison with Proposition 4 of [B, Ch.IV, $\S 5]$ that the set

$$
\left\{\left(e_{i_{1}}^{(0)}, \ldots, e_{i_{s}}^{(0)} ; I\right)_{0} ;|I|=k \text { and } 1 \leq i_{1}<\cdots<i_{s} \leq m\right\}
$$

is a basis of $\Gamma^{k} M_{0}$. It is also not difficult to verify that

$$
\left\{\left(e_{j_{1}}^{(1)}, \ldots, e_{j_{l}}^{(1)}\right)_{1} ; 1 \leq j_{1}<\cdots<j_{l} \leq n\right\}
$$

is a basis of $\Gamma^{l} M_{1}$. The lemma then follows from (26).

We are now ready to prove the main theorem.

Theorem 4.2. Assume $m, n \geq d$. Then evaluation on $\mathbb{k}^{m \mid n}, \mathcal{U}(1)^{\oplus n}$ yields equivalences of categories:

$$
\mathrm{Pol}_{d}^{(\mathrm{I})} \stackrel{\sim}{\longrightarrow} \mathcal{S}(m \mid n, d) \text { smod, } \quad \mathrm{Pol}_{d}^{(\mathrm{II})} \stackrel{\sim}{\longrightarrow} \mathcal{Q}(n, d) \text { smod }
$$

respectively. 
Proof. We prove only the second equivalence, since the proof of the first equivalence is similar. Recall that

$$
\mathcal{Q}(n, d)=\operatorname{end}_{\Gamma_{Q}^{d}}\left(\mathcal{U}(1)^{\oplus n}\right) .
$$

According to Proposition A.1, it suffices to show that the map induced by composition,

$$
\operatorname{hom}_{\Gamma_{Q}^{d}}\left(\mathcal{U}(1)^{\oplus n}, W\right) \otimes \operatorname{hom}_{\Gamma_{Q}^{d}}\left(V, \mathcal{U}(1)^{\oplus n}\right) \rightarrow \operatorname{hom}_{\Gamma_{Q}^{d}}(V, W)
$$

is surjective for all $V, W \in \Gamma_{Q}^{d}$. It follows from Example 2.6 that for any $r \in \mathbb{Z}_{2}$ there exist bases $\left(x^{(r)}(j, i)\right),\left(y^{(r)}(k, j)\right)$ and $\left(z^{(r)}(k, i)\right)$ of $\operatorname{Hom}_{\mathcal{C}(1)}\left(V, \mathcal{U}(1)^{\oplus n}\right)_{r}$, $\operatorname{Hom}_{\mathcal{C}(1)}\left(\mathcal{U}(1)^{\oplus n}, W\right)_{r}$ and $\operatorname{Hom}_{\mathcal{C}(1)}(V, W)_{r}$ respectively, such that

$$
y^{(r)}\left(k, j_{1}\right) \circ x^{\left(r^{\prime}\right)}\left(j_{2}, i\right)=\delta_{j_{1}, j_{2}} z^{\left(r+r^{\prime}\right)}(k, i),
$$

for $r, r^{\prime} \in \mathbb{Z}_{2}$, where $\delta_{j_{1}, j_{2}}$ is the Kronecker delta.

To prove surjectivity, it suffices to show for $1 \leq s, t \leq d$ that each element of the form

$$
\left(z^{(0)}\left(k_{1}, i_{1}\right), \ldots, z^{(0)}\left(k_{s}, i_{s}\right) ; I\right)_{0} \cdot\left(z^{(1)}\left(k_{1}^{\prime}, i_{1}^{\prime}\right), \ldots, z^{(1)}\left(k_{t}^{\prime}, i_{t}^{\prime}\right)\right)_{1}
$$

in $\Gamma^{d} \operatorname{Hom}_{\mathcal{C}(1)}(V, W) \simeq \operatorname{hom}_{\Gamma_{Q}^{d}}(V, W)$ lies in the image of (27)), since $\Gamma^{d} \operatorname{Hom}_{\mathcal{C}(1)}(V, W)$ is spanned by such elements according to Lemma 4.1. Suppose $I=\left(d_{1}, \ldots, d_{s}\right) \in$ $\left(\mathbb{Z}_{>0}\right)^{s}$. Then, since $n \geq d=|I|+t \geq s+t$, we may choose distinct indices $j_{1}, \ldots, j_{s}, j_{1}^{\prime}, \ldots, j_{t}^{\prime}$ to form the element

$$
\begin{aligned}
& \left(y^{(0)}\left(k_{1}, j_{1}\right), \ldots, y^{(0)}\left(k_{s}, j_{s}\right) ; I\right)_{0} \cdot\left(y^{(1)}\left(k_{1}^{\prime}, j_{1}^{\prime}\right), \ldots, y^{(1)}\left(k_{t}^{\prime}, j_{t}^{\prime}\right)\right)_{1} \\
& \otimes\left(x^{(0)}\left(j_{1}, i_{1}\right), \ldots, x^{(0)}\left(j_{s}, i_{s}\right), x^{(0)}\left(j_{1}^{\prime}, i_{1}^{\prime}\right), \ldots, x^{(0)}\left(j_{t}^{\prime}, i_{t}^{\prime}\right) ; J\right)_{0},
\end{aligned}
$$

where $J=\left(d_{1}, \ldots, d_{s}, 1, \ldots, 1\right) \in\left(\mathbb{Z}_{>0}\right)^{s+t}$, which is sent to the element (28) under the map induced by composition in $\Gamma_{Q}^{d}$.

From the previous thereom and the classifications given in [D, BrK1 we obtain the following corollary. By a partition we mean an infinite nonincreasing sequence $\lambda=\left(\lambda_{1}, \lambda_{2}, \ldots\right)$ of nonnegative integers such that the sum $|\lambda|=\sum \lambda_{i}$ is finite. Let $\mathscr{P}$ denote the set of all partitions.

Corollary 4.3. If $p>2$, then the set of distinct isomorphism classes of simple objects of $\mathrm{Pol}_{d}^{(\mathrm{I})}$ is in bijective correspondence with the set of pairs

$$
\{(\lambda, \mu) ; \lambda, \mu \in \mathscr{P} \text { and }|\lambda|+p|\mu|=d\} .
$$

On the other hand, if $p=0$, the classes of simple objects correspond with $\{\lambda \in$ $\mathscr{P} ;|\lambda|=d\}$.

Now suppose in addition that the field $\mathbb{k}$ is algebraically closed. Then the set of classes of simple objects of $\mathrm{Pol}_{d}^{(\mathrm{II})}$ is in bijective correspondence with the set of partitions

$$
\left\{\lambda \in \mathscr{P} ;|\lambda|=d \text {, and } \lambda_{i} \neq \lambda_{i+1} \text { if } p \nmid \lambda_{i}\right\} \text {. }
$$


4.2. Spin polynomial functors and Sergeev duality. In this section, assume the ground field $\mathbb{k}$ is algebraically closed. We limit our attention to the objects $T \in \mathrm{Pol}_{d}^{(\mathrm{II})}$, which may be called spin polynomial functors. The explanation for this term is given by Theorem 4.4 below, which describes a relationship between $\mathrm{Pol}_{d}^{(\mathrm{II})}$ and finite dimensional representations of the Sergeev superalgebra, which is "super equivalent" to the spin symmetric group algebra $\mathbb{k} \mathfrak{S}_{d}^{-}$(cf. [BrK1]).

The (external) tensor product defined in Section 2.5 induces a bifunctor

$$
-\otimes-: \mathrm{Pol}_{d}^{(\mathrm{II})} \times \mathrm{Pol}_{e}^{(\mathrm{II})} \rightarrow \mathrm{Pol}_{d+e}^{(\mathrm{II})} .
$$

Let us consider the category: Pol $^{(\mathrm{II})}=\bigoplus_{d \geq 0} \mathrm{Pol}_{d}^{(\mathrm{II})}$. We then have an induced bifunctor

$$
-\otimes-: \mathrm{Pol}^{(\mathrm{II})} \times \mathrm{Pol}^{(\mathrm{II})} \rightarrow \mathrm{Pol}^{(\mathrm{II})},
$$

which gives $\mathrm{Pol}^{(\mathrm{II})}$ the structure of a monoidal category.

Suppose now $M, N \in \operatorname{svec}_{\mathbb{k}}$. Then $\Gamma^{*}()$ satisfies the exponential property

$$
\Gamma^{*}(M \oplus N) \cong \Gamma^{*} M \otimes \Gamma^{*} N,
$$

which follows from (44) and the corresponding properties for $D^{*}()$ and $\Lambda^{*}()$. It follows from (26) and (29) that

$$
\Gamma^{d}(M \oplus N)=\bigoplus_{i=0}^{d} \Gamma^{d-i} M \otimes \Gamma^{i} N .
$$

Recall the objects $\Gamma^{d, n} \in$ Pol $_{d}^{(\mathrm{II})}$ which are projective by Yoneda's lemma (see Section 3). It follows from (30) that we have a decomposition

$$
\Gamma^{d, m+n} \simeq \bigoplus_{i+j=d} \Gamma^{i, m} \otimes \Gamma^{j, n}
$$

of strict polynomial functors.

Now let $\Lambda(n, d)$ denote the set of all tuples $\lambda=\left(\lambda_{1}, \ldots, \lambda_{n}\right) \in\left(\mathbb{Z}_{>0}\right)^{n}$ such that $\sum \lambda_{i}=d$. Given $\lambda \in \Lambda(n, d)$, we will write $\Gamma^{\lambda}=\Gamma^{\lambda_{1}, 1} \otimes \cdots \otimes \Gamma^{\lambda_{n}, 1}$. By (31) and induction, we have a canonical isomorphism

$$
\Gamma^{d, n} \simeq \bigoplus_{\lambda \in \Lambda(n, d)} \Gamma^{\lambda}
$$

It follows that the objects $\Gamma^{\lambda}$ are projective in $\mathrm{Pol}_{d}^{(\mathrm{II})}$.

Let $\omega=(1, \ldots, 1) \in \Lambda(d, d)$. Then $\Gamma^{\omega}=\otimes^{d}$, and $\otimes^{d}$ is a projective object of $\mathrm{Pol}_{d}^{(\mathrm{II})}$. We have the following analogue of [BrK1, Theorem 6.2].

Theorem 4.4. Assume $n \geq d$.

(i) The left $\mathcal{Q}(n, d)$-supermodule $V^{\otimes d} \simeq \operatorname{hom}_{\mathrm{Pol}_{d}^{(\mathrm{II})}}\left(\Gamma^{d, n}, \otimes^{d}\right)$ is a projective object of $\mathcal{Q}(n, d)$ smod.

(ii) There is a canonical isomorphism of superalgebras

$$
\operatorname{end}_{\mathrm{Pol}_{d}^{(\mathrm{II})}}\left(\otimes^{d}\right) \cong \mathcal{W}(d) \text {. }
$$

(iii) We have an exact functor

$$
\operatorname{hom}_{\mathrm{Pol}_{d}^{(\mathrm{II})}}\left(\otimes^{d},-\right): \mathrm{Pol}_{d}^{(\mathrm{II})} \rightarrow \mathcal{W}(d) \text { smod. }
$$


Proof. (i) follows from Theorem 4.2 and the fact that $\bigotimes^{d}$ is a projective object of $\mathrm{Pol}_{d}^{(\mathrm{II})}$, and (ii) follows from Theorem 4.2 and [BrK1, Theorem 6.2.(iii)]. Finally, (iii) is a direct consequence of (i) and (ii).

Remark 4.5. One may refer to the functor in Theorem 4.4.(iii) as the Sergeev duality functor. A similar functor related to classical Schur-Weyl duality was studied in $[\mathrm{HY}]$ in the context of $\mathfrak{g}$-categorification.

\section{CATEGORIES OF $\mathfrak{s i c h} \mathfrak{k}_{\mathbb{k}}$-ENRICHED FUNCTORS}

In this section, we provide an alternate definition of strict polynomial functors which is a "super analogue" of Friedlander and Suslin's original definition [FS, Definition 2.1]. We also introduce categories $\mathfrak{P o r}_{d}^{(\mathrm{I})}$ and $\mathfrak{P o r}_{d}^{(\mathrm{II})}$ whose objects are homogeneous $\mathfrak{s s c h}_{\mathbb{K}_{\mathbb{k}}}$-enriched functors between a pair of $\mathfrak{s s c h}_{\mathbb{R}^{-}}$-enriched categories. Familiarity with the notation and material from Appendix B will be assumed throughout this section.

5.1. Definition of $\mathfrak{s s c h}_{\mathbb{k}}$-enriched functors. Recall that we may identify $\mathfrak{s s c h}_{\mathbb{k}}$ as a full subcategory of the functor category $\operatorname{Fct}\left(\mathfrak{s a l} \mathfrak{g}_{\mathfrak{k}}, \mathfrak{s e t s}\right)$. Given superschemes $X, Y \in \mathfrak{s s c h}_{\mathbb{k}}$, the functor $X \times Y$ is again a superscheme. Let $I_{0}$ be a constant functor such that $I_{0}(\mathcal{A})=\{0\}$ for all $\mathcal{A} \in \mathfrak{s a l g}_{\mathfrak{k}}$. Then $I_{0}$ is an affine superscheme with $\mathbb{k}\left[I_{0}\right]=\mathbb{k}$. The monoidal structure on the category $\mathfrak{s e t s}$ with respect to direct product induces a corresponding (symmetric) monoidal structure on $\mathfrak{s s c h}_{\mathbb{k}}$, such that $I_{0}$ is an identity element.

Let $X, Y \in \mathfrak{s s c h}_{\mathbb{k}}$, with $X$ an affine superscheme. An analogue of [Jan, I.1.3] (Yoneda's lemma for ordinary schemes) gives a bijection

$$
\operatorname{hom}_{\mathfrak{s} \mathfrak{s c h}_{\mathbb{k}}}(X, Y) \stackrel{\sim}{\longrightarrow} Y(\mathbb{k}[X]) .
$$

Let $\mathcal{B}$ an associative superalgebra, and suppose $U, V, W \in{ }_{\mathcal{B}}$ smod. Then, there is a natural transformation

$$
\operatorname{Hom}_{\mathcal{B}}(V, W)_{a} \times \operatorname{Hom}_{\mathcal{B}}(U, V)_{a} \rightarrow \operatorname{Hom}_{\mathcal{B}}(U, W)_{a}
$$

given by the isomorphism (36) and composition of $\mathcal{A}$-linear maps, for all $\mathcal{A} \in \mathfrak{s a l g}_{\mathbb{k}}$. We also have for each $V \in \operatorname{svec}_{\mathcal{B}}$ a natural transformation

$$
j_{V}: I_{0} \rightarrow \operatorname{End}_{\mathcal{B}}(V)_{a}
$$

which is the element of $\operatorname{hom}_{\mathfrak{s s c h}}\left(I_{0}, \operatorname{End}_{\mathcal{B}}(V)_{a}\right)$ mapped onto $\operatorname{Id}_{V} \in \operatorname{End}_{\mathcal{B}}(V)_{0}$ under the bijection (33). It then may be checked that we obtain an $\mathfrak{s s c h}_{\mathbb{K}^{-}}$-enriched category $\mathcal{B} \mathfrak{m o d}$ (in the sense of $[\mathrm{Ke}]$ ) which has the same objects as $\mathcal{B}$ smod and hom-objects

$$
\operatorname{hom}_{\mathcal{B} \mathfrak{s m o d}}(V, W)=\operatorname{Hom}_{\mathcal{B}}(V, W)_{a}
$$

for all $V, W \in{ }_{\mathcal{B}}$ smod. If $\mathcal{B}=\mathbb{k}$, we write $\mathfrak{B}_{\mathfrak{B m o d}}=\mathfrak{s} \mathfrak{v} \mathfrak{c}_{\mathbb{k}}$.

Definition 5.1. Suppose $\mathcal{B}$ is an associative superalgebra. Let $\mathcal{V}={ }_{\mathcal{B}}$ smod, and let $\mathfrak{V}$ denote the corresponding $\mathfrak{s} \mathfrak{s c h} \mathfrak{k}_{\mathbb{k}}$-enriched category. A $\mathfrak{s s c h}_{\mathbb{k}}$-enriched functor $\left(\right.$ or $\mathfrak{s s c h}_{\mathbb{k}}-$ functor $)$

$$
T: \mathfrak{V} \rightarrow \mathfrak{s v e c}_{\mathbb{k}}
$$

consists of an assignment

$$
T(V) \in \operatorname{svec}_{\mathbb{k}} \quad\left({ }^{\forall} V \in \mathcal{V}\right)
$$


and a morphism of superschemes

$$
T_{V, W}: \operatorname{Hom}_{\mathcal{B}}(V, W)_{a} \rightarrow \operatorname{Hom}_{\mathbb{k}}(T(V), T(W))_{a} \quad\left({ }^{\forall} V, W \in \mathcal{V}\right),
$$

such that the following two diagrams commute for all $U, V, W \in \mathcal{V}$ :

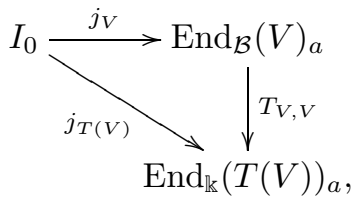

and

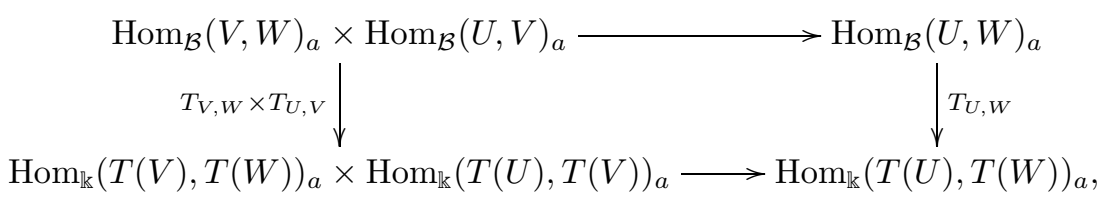

with horizontal maps being given by composition in $\mathfrak{V}$ and $\mathfrak{s} \mathfrak{v} \mathfrak{c}_{\mathbb{k}}$, respectively.

5.2. The categories $\mathfrak{P o l}_{d}^{(\dagger)}$ for $\dagger=$ I, II. Notice that if $f: M \rightarrow N$ is an even linear map of vector superspaces, then $f$ may be identified with the associated natural transformation $\eta_{f}: M_{a} \rightarrow N_{a}$ which is given by the $\mathbb{k}$-linear maps

$$
\eta_{f}(\mathcal{A})=f \otimes 1_{\mathcal{A}}:(M \otimes \mathcal{A})_{0} \rightarrow(N \otimes \mathcal{A})_{0},
$$

for all $\mathcal{A} \in \mathfrak{s a l g}_{\mathfrak{k}}$.

Definition 5.2. Let $\mathcal{V}={ }_{\mathcal{B}}$ smod, and let $\mathfrak{V}={ }_{\mathcal{B}} \mathfrak{s m o d}$. Suppose that $S, T: \mathfrak{V} \rightarrow$ $\mathfrak{s v e c} \mathfrak{c}_{\mathbb{k}}$ are both $\mathfrak{s} \mathfrak{s c h} \mathfrak{K}_{\mathbb{k}}$-functors. Then a $\mathfrak{s s c h}_{\mathbb{K}_{\mathbb{k}}}$-natural transformation, $\alpha: S \rightarrow T$, is defined to be a collection of even $\mathbb{k}$-linear maps $\alpha_{V}: S(V) \rightarrow T(V)$ such that the following diagram commutes for all $V, W \in \mathfrak{V}$ :

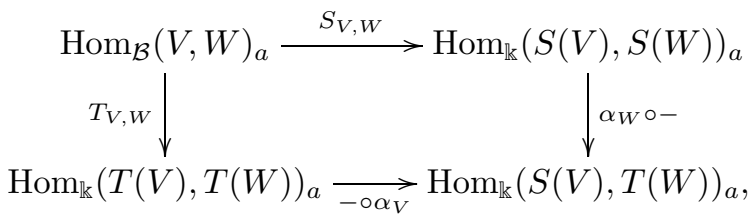

where we have identified the even linear maps, $\alpha_{W} \circ-$ and $-\circ \alpha_{V}$, with their corresponding natural transformations as described in the preceding paragraph. Denote by Fet $_{\mathfrak{s s c h}_{\mathfrak{k}}}\left(\mathfrak{V}, \mathfrak{s v e c}_{\mathbb{k}}\right)$ the category of all $\mathfrak{s s c h}_{\mathbb{k}^{\mathrm{k}}}-$ functors, $T: \mathfrak{V} \rightarrow \mathfrak{s v e c}_{\mathbb{k}}$, and $\mathfrak{s i c h} \mathfrak{k}_{\mathbb{k}}$-natural transformations.

Let $\mathfrak{V}={ }_{\mathcal{B}} \mathfrak{s m o d}$, and suppose $V \in \mathcal{B}_{\text {smod. }}$ Given $T \in \mathrm{Fct}_{\mathfrak{s i c h} \mathfrak{l}_{\mathbb{k}}}\left(\mathfrak{V}, \mathfrak{s v e c}_{\mathbb{k}}\right)$ consider the algebraic supergroup $G=G L_{\mathcal{B}, V}$ and recall that $\operatorname{End}_{B, V}=\operatorname{End}_{\mathcal{B}}(V)_{a}$. Then, by the definition of $\mathfrak{s s c h} \mathfrak{\mathbb { R }}_{\mathbb{k}}$ functor, the induced natural transformation $T_{V, V}$ : $E n d_{\mathcal{B}, V} \rightarrow E n d_{\mathbb{k}, T(V)}$ restricts to a natural transformation of supergroups,

$$
\eta^{T, V}: G \rightarrow G L_{\mathbb{k}, T(V)},
$$

which preserves identity and products. Hence $\eta^{T, V}$ is a representation of the supergroup $G$.

Now $T(V)$ may also be considered as a $G$-supermodule with a corresponding structure map

$$
\Delta_{T, V}: T(V) \rightarrow T(V) \otimes \mathbb{k}[G]
$$


Notice that for any $M, N \in \mathrm{svec}_{\mathbb{k}}$, Yoneda's lemma gives a canonical isomorphism

$$
\operatorname{hom}_{\mathfrak{s s c h} \mathfrak{k}_{\mathrm{k}}}\left(M_{a}, N_{a}\right) \simeq\left(N \otimes \mathbb{k}\left[M_{a}\right]\right)_{0}
$$

for the corresponding affine superschemes. Using (34), let us identify the natural transformation $T_{V, V}$ with an element of the set

$$
\left(\operatorname{End}_{\mathbb{k}}(T(V)) \otimes \mathbb{k}\left[\operatorname{end}_{\mathcal{V}}(V)_{a}\right]\right)_{0} .
$$

It is then not difficult to see how $T_{V, V}$ gives rise to the structure map $\Delta_{T, V}$. Hence the image of $\Delta_{T, V}$ lies in $T(V) \otimes \mathbb{k}\left[E n d_{\mathcal{B}, V}\right]$, and $T(V)$ is a polynomial representation of $G$.

Definition 5.3. Let $\mathfrak{V}={ }_{\mathcal{B}} \mathfrak{s m o d}$. We define $\operatorname{Fct}_{\mathfrak{s s c h}}\left(\mathfrak{V}, \mathfrak{s v e c}_{\mathbb{k}}\right)_{(d)}$ to be the full subcategory of $\mathrm{Fct}_{\mathfrak{s} \mathfrak{s c h}}\left(\mathfrak{V}, \mathfrak{s v e c}_{\mathbb{k}}\right)$ consisting of all $\mathfrak{s s c h}_{\mathbb{k}^{-}}$-enriched functors $T: \mathfrak{V} \rightarrow$ $\mathfrak{s} \mathfrak{v e c} \mathfrak{c}_{\mathbb{k}}$ such that

$$
T_{V, W} \in\left(\operatorname{Hom}_{\mathbb{k}}(T(V), T(W)) \otimes S^{d}\left(\operatorname{Hom}_{\mathcal{B}}(V, W)^{\vee}\right)_{0}\right.
$$

for all $V, W \in \mathcal{B}_{\mathcal{B}}$ smod (where we have identified both sides of (34)). We write

$$
\begin{aligned}
& \mathfrak{P o r}_{d}^{(\mathrm{I})}=\operatorname{Fct}_{\mathfrak{s s c h}_{\mathfrak{k}}}\left(\mathfrak{s v e c}_{\mathbb{k}}, \mathfrak{s v e c _ { \mathbb { k } }}\right)_{(d)} \text {, and } \\
& \mathfrak{P o r} \mathfrak{l}_{d}^{(\mathrm{II})}=\operatorname{Fct}_{\mathfrak{s s c h} \mathfrak{k}_{\mathrm{k}}}\left(\mathcal{C}(1) \mathfrak{s m o d}, \mathfrak{s v e c} \mathfrak{c}_{\mathbb{k}}\right)_{(d)} \text {. }
\end{aligned}
$$

From Theorem 5.4 below, it follows that these categories are equivalent to Pol ${ }_{d}^{(\mathrm{I})}$ and $\mathrm{Pol}_{d}^{(\mathrm{II})}$ respectively.

5.3. Polynomial representations of $G L(m \mid n)$ and $Q(n)$. Suppose $m, n$ are fixed nonnegative integers. Let us write $\mathcal{S}^{\mathrm{I}}=\mathcal{S}(m \mid n, d)$ and $\mathcal{S}^{\mathrm{II}}=\mathcal{Q}(n, d)$. We also write $G^{\mathrm{I}}=G L(m \mid n)$ and $G^{\mathrm{II}}=Q(n)$. If $\dagger=\mathrm{I}$, let $V_{l}=V_{r}=\mathbb{k}^{m \mid n} \in \operatorname{svec}_{\mathbb{k}}$, and if $\dagger=\mathrm{II}$, let $V_{l}=\mathcal{U}_{l}(1)^{\oplus n} \in \mathcal{C}(1)$ smod and $V_{r}=\mathcal{U}(1)^{\oplus n} \in \operatorname{smod}_{\mathcal{C}(1)}$.

Theorem 5.4. Suppose $m, n \geq d$. Then we have equivalences of categories:

$$
\text { (i) } \Psi: \mathfrak{p o l}_{d}\left(G^{\dagger}\right) \stackrel{\sim}{\longrightarrow} \mathcal{S}^{\dagger} \text { smod, } \quad \text { (ii) } \Phi: \mathfrak{P o r}_{d}^{(\dagger)} \stackrel{\sim}{\longrightarrow} \operatorname{Pol}_{d}^{(\dagger)},
$$

for $\dagger=I$, II respectively

Proof. Proof of (i). Let $\mathcal{B}=\mathbb{k}, \mathcal{C}(1)$ if $\dagger=\mathrm{I}$, II respectively. It suffices to show that we have an isomorphism of superalgebras

$$
\mathcal{S}^{\dagger} \cong\left(\mathbb{k}\left[\operatorname{End}_{\mathcal{B}}\left(V_{l}\right)\right]_{d}\right)^{\vee} \text {. }
$$

Using Proposition B.1.(iii), (8), (7) and (12), we have

$$
\begin{aligned}
\left(\mathbb{k}\left[\operatorname{End}_{\mathcal{B}, V_{l}}\right]_{d}\right)^{\vee} & =S^{d}\left(\operatorname{End}_{\mathcal{B}}\left(V_{l}\right)^{\vee}\right)^{\vee} \\
& \cong \Gamma^{d}\left(\operatorname{End}_{\mathcal{B}}\left(V_{l}\right)^{-}\right) \cong \Gamma^{d}\left(\operatorname{End}_{\mathcal{B}}\left(V_{r}\right)\right)=\mathcal{S}^{\dagger} .
\end{aligned}
$$

Proof of (ii). Let $\mathcal{V}={ }_{\mathcal{B}}$ smod for $\mathcal{B}$ as above. Then we identify $\mathcal{V}^{-}$with $\operatorname{svec}_{\mathbb{k}}, \operatorname{smod}_{\mathcal{C}(1)}$ respectively, using (77) and (12). Hence the objects of $\mathcal{V}$ are identical to the objects of either $\mathcal{V}^{-}$or $\Gamma^{d}\left(\mathcal{V}^{-}\right)$respectively.

Suppose $T \in \mathfrak{P o l}_{d}^{(\dagger)}$. We will define a functor $\Phi(T): \Gamma^{d}\left(\mathcal{V}^{-}\right) \rightarrow \operatorname{svec}_{\mathbb{k}}$. Given $V \in \mathcal{V}$, let $\Phi(T)(V)=T(V) \in \operatorname{svec}_{\mathbb{k}}$. Now suppose $V, W \in \mathcal{V}^{-}$. We have a map

$$
\begin{aligned}
T_{V, W} & \in S^{d}\left(\operatorname{hom}_{\mathcal{V}}(V, W)^{\vee}\right) \otimes \operatorname{Hom}(T(V), T(W))_{0} \\
& \cong \operatorname{Hom}\left(\operatorname{Hom}(T(V), T(W))^{\vee}, S^{d}\left(\operatorname{hom}_{\mathcal{V}}(V, W)^{\vee}\right)\right)_{0} \\
& \cong \operatorname{Hom}\left(\Gamma^{d} \operatorname{hom}_{\mathcal{V}^{-}}(V, W), \operatorname{Hom}(T(V), T(W))\right)_{0} .
\end{aligned}
$$


Let $\Phi(T)_{V, W}: \operatorname{hom}_{\Gamma^{d}\left(\mathcal{V}^{-}\right)}(V, W) \rightarrow \operatorname{Hom}(T(V), T(W))$ denote the image of $T_{V, W}$ under the above isomorphism. Then it may be checked that $\Phi(T) \in \operatorname{Fct}\left(\Gamma^{d}\left(\mathcal{V}^{-}\right)\right.$, $\mathrm{svec}_{\mathbb{k}}$ ), and that this gives an equivalence of categories

$$
\Phi: \mathfrak{P o l}_{d}^{(\dagger)} \stackrel{\sim}{\longrightarrow} \mathrm{Pol}_{d}^{(\dagger)}
$$

which maps $T \mapsto \Phi(T)$.

Corollary 5.5. Suppose $m, n \geq d$, and let $V_{l}, V_{r}$ be as above. Then we have a commutative diagram

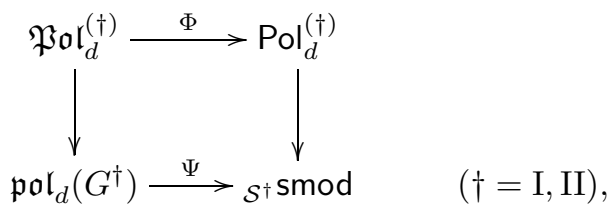

where the vertical arrow on the left is evaluation at $V_{l}$ and the vertical arrow on the right is evaluation at $V_{r}$. In particular, evaluation at $V_{l}$ gives an equivalence $\mathfrak{P o r}_{d}^{(\dagger)} \stackrel{\sim}{\longrightarrow} \mathfrak{p o l}_{d}\left(G^{\dagger}\right)$ for $\dagger=I$, II respectively.

Proof. We know that the vertical arrow on the left is an equivalence by Theorem 4.2. It is then not difficult to see from the definitions of the functors $\Phi$ and $\Psi$ that the diagram is commutative. Hence, from Theorem 5.4 the commutativity implies that the evaluation at $V_{r}$ also gives an equivalence.

\section{ApPendix A. RePRESEntATIONS OF svec $_{\mathbb{k}}$-ENRICHED CATEGORIES}

Recall that $\mathbb{k}$ is a field of characteristic not equal 2 , and $\mathbf{s v e c}_{\mathbb{k}}$ denotes the category of finite dimensional vector superspaces over $\mathbb{k}$. Suppose $\mathcal{V}$ is a category enriched over $\mathrm{svec}_{\mathbb{k}}$. In this appendix we describe the relationship between the following two categories:

(i) The category $\mathcal{V}$-smod $=\mathrm{Fct}_{\mathbb{k}}\left(\mathcal{V}\right.$, $\left.\operatorname{svec}_{\mathbb{k}}\right)$ of all $\mathbb{k}$-linear representations of $\mathcal{V}$. It consists of all even $\mathbb{k}$-linear functors, $\mathcal{V} \rightarrow \mathrm{svec}_{\mathbb{k}}$, and $\mathrm{svec}_{\mathbb{k}^{-}}$-enriched natural transformations (see Section 2.6).

(ii) If $P \in \mathcal{V}$, then $\mathcal{E}=\operatorname{End}_{\mathcal{V}}(P)$ is an associative superalgebra with product given by composition. We may then consider the category $\mathcal{E}$ smod of finite dimensional left supermodules over $\mathcal{E}$.

The categories $\mathcal{V}$-smod and $\mathcal{E}_{\mathcal{S}}$ smod are both $\mathrm{svec}_{\mathbb{k}_{\mathrm{k}}}$-enriched categories. We denote by $(\mathcal{V} \text {-smod })_{\text {ev }},(\mathcal{E} \text { smod })_{\text {ev }}$ the corresponding even subcategories. Recall from Section 2 that $\left(\mathcal{A}_{\mathrm{smod}}\right)_{\mathrm{ev}}$ is an abelian category for any finite dimensional superalgebra $\mathcal{A}$. In particular, $\left({ }_{\mathcal{E}} \mathrm{smod}\right)_{\mathrm{ev}}$ and $\left(\mathrm{svec}_{\mathbb{k}}\right)_{\mathrm{ev}}$ are both abelian categories. Now since direct sums, products, kernels and cokernels can be computed objectwise in (the even subcategory of) the target category $\mathrm{svec}_{\mathbb{k}}$, we see that $(\mathcal{V} \text {-smod })_{\mathrm{ev}}$ is also an abelian category.

The relationship between $\mathcal{V}$-smod and $\mathcal{E}_{\mathcal{E}}$ smod is given by evaluation on $P$. If $F \in \mathcal{V}$-smod, the (even) functoriality of $F$ makes the $\mathbb{k}$-superspace $F(P)$ into a supermodule over $\mathcal{E}=\operatorname{end}_{\mathcal{V}}(P)$. We thus have an evaluation functor:

$$
\begin{gathered}
\mathcal{V} \text {-smod } \rightarrow{ }_{\mathcal{E} \text { smod }}, \\
F \mapsto F(P) .
\end{gathered}
$$


There is another interpretation of this evaluation functor. Since the covariant homfunctor $h^{P}:=\operatorname{hom}_{\mathcal{V}}(P,-)$ is an even $\mathbb{k}$-linear functor, it must belong to $\mathcal{V}$-smod. In this situation, Yoneda's lemma takes the form of an even isomorphism

$$
\operatorname{hom}_{\mathcal{V} \text {-smod }}\left(h^{P}, F\right) \simeq F(P),
$$

for any $F \in \mathcal{V}$-smod. In particular,

$$
\mathcal{E}=h^{P}(P) \simeq \operatorname{end} \mathcal{V}_{\text {-smod }}\left(h^{P}\right) .
$$

Hence, Yoneda's lemma allows us to interpret "evaluation at $P$ " as the functor $\operatorname{hom}_{\mathcal{V} \text {-smod }}\left(h^{P},-\right): \mathcal{V}$-smod $\rightarrow \mathcal{E}^{\text {smod. }}$

We are interested to know if there is some condition on $P$ which ensures that evaluation is in fact an equivalence of categories. The next proposition, which is a super analogue of [T2, Prop. 7.1], provides such a criterion.

Note that the parity change functor, $\Pi: \mathrm{svec}_{\mathbb{k}} \rightarrow \mathrm{svec}_{\mathbb{k}}$, induces by composition a functor $\Pi \circ-: \mathcal{V}$-smod $\rightarrow \mathcal{V}$-smod.

Proposition A.1. Let $\mathcal{V}$ be an $\mathrm{svec}_{\mathbb{k}_{\mathrm{k}}}$-enriched category. Assume that there exists an object $P \in \mathcal{V}$ such that for all $X, Y \in \mathcal{V}$, the composition induces a surjective map

$$
\operatorname{hom}_{\mathcal{V}}(P, Y) \otimes \operatorname{hom}_{\mathcal{V}}(X, P) \rightarrow \operatorname{hom}_{\mathcal{V}}(X, Y) .
$$

Then the following hold.

(i) For all $F \in \mathcal{V}$-smod and all $Y \in \mathcal{V}$, the canonical map $\operatorname{hom}_{\mathcal{V}}(P, Y) \otimes$ $F(P) \rightarrow F(Y)$ is surjective.

(ii) The set $\left\{h^{P}, \Pi h^{P}\right\}$ is a projective generator of $(\mathcal{V} \text {-smod })_{\mathrm{ev}}$, where $h^{P}=$ $\operatorname{hom}_{\mathcal{V}}(P,-)$ as above.

(iii) Let $\mathcal{E}=\operatorname{end}_{\mathcal{V}}(P)$. Then evaluation on $P$ induces an equivalence of categories $\mathcal{V}$-smod $\simeq \mathcal{E}$ smod.

Proof. Proof of (i). The canonical map is: $f \otimes x \mapsto F(f)(x)$. By the surjectivity of $\operatorname{hom}_{\mathcal{V}}(P, Y) \otimes \operatorname{hom}_{\mathcal{V}}(Y, P) \rightarrow \operatorname{hom}_{\mathcal{V}}(Y, Y)$, we may find a finite family of maps, $\alpha_{i} \in \operatorname{hom}_{\mathcal{V}}(P, Y)$ and $\beta_{i} \in \operatorname{hom}_{\mathcal{V}}(Y, P)$, such that

$$
\sum_{i} \alpha_{i} \circ \beta_{i}=\operatorname{Id}_{Y}
$$

Now suppose that $y \in F(Y)$. Then one may check that the element

$$
\sum_{i} \alpha_{i} \otimes F\left(\beta_{i}\right)(y) \in \operatorname{hom}_{\mathcal{V}}(P, Y) \otimes F(P)
$$

is sent onto $y$ by the canonical map.

Proof of (ii). The Yoneda isomorphism hom $\mathcal{V}_{\text {-smod }}\left(h^{P}, F\right) \simeq F(P)$ ensures that $h^{P}$ is projective. One may check that $\Pi h^{P}$ is then also a projective object of $(\mathcal{V} \text {-smod })_{\text {ev }}$. Next, by the naturality of the canonical map, (i) yields an epimorphism $h^{P} \otimes F(P) \rightarrow F$. Now $F(P)$ is a finite dimensional superspace. By choosing a $\left(\mathbb{Z}_{2}\right.$-homogeneous) basis of $F(P)$, we have $F(P) \simeq \mathbb{k}^{m \mid n}$ where $\operatorname{sdim}(P)=(m, n)$. Hence, there exists an epimorphism $\varphi:\left(h^{P}\right)^{\oplus m} \oplus\left(\Pi h^{P}\right)^{\oplus n} \rightarrow F$, and we may write $\varphi=\varphi_{1}+\cdots+\varphi_{m}+\varphi_{1}^{\prime}+\cdots+\varphi_{n}^{\prime}$ for some $\varphi_{i}: h^{P} \rightarrow F\left(\right.$ resp. $\left.\varphi_{j}^{\prime}: \Pi h^{P} \rightarrow F\right)$, where $i=1, \ldots, m$ (resp. $j=1, \ldots, n)$. Then we may finally decompose

$$
F=\bigoplus_{i=1}^{m} F_{i} \oplus \bigoplus_{j=1}^{n} F_{j}^{\prime},
$$


where $F_{i}=\operatorname{Im}\left(\varphi_{i}\right)$ (resp. $\left.F_{j}^{\prime}=\operatorname{Im}\left(\varphi_{j}^{\prime}\right)\right)$. It then follows that $\left\{h^{P}, \Pi h^{P}\right\}$ is a generating set.

Proof of (iii). We first verify that evaluation is fully faithful. For this purpose, it suffices to check for any $F, G \in \mathcal{V}$-smod that we have an isomorphism: $\operatorname{hom}_{\mathcal{V} \text {-smod }}(G, F) \simeq \operatorname{Hom}_{\mathcal{E}}(G(P), F(P))$. Notice that there is a commutative triangle:

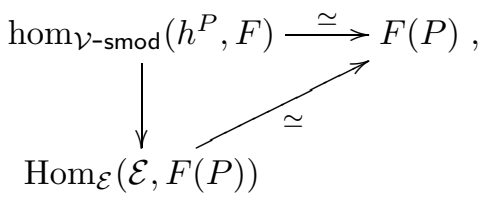

where the horizontal arrow is the Yoneda isomorphism, and the diagonal arrow is the isomorphism (9) from Section2. Hence the diagram induces an (even) isomorphism. By additivity of homs, we also have an isomorphism

$$
\operatorname{hom}_{\mathcal{V} \text {-smod }}\left(h^{p} \otimes \mathbb{k}^{m \mid n}, F\right) \simeq \operatorname{Hom}_{\mathcal{E}}\left(\mathcal{E} \otimes \mathbb{k}^{m \mid n}, F(P)\right),
$$

for any $m, n \in \mathbb{N}$. Now by (ii) we may find (for any $G \in \mathcal{V}$-smod) an exact sequence

$$
h^{P} \otimes \mathbb{k}^{m_{2} \mid n_{2}} \rightarrow h^{P} \otimes \mathbb{k}^{m_{1} \mid n_{1}} \rightarrow G \rightarrow 0 .
$$

It then follows by the left exactness of $\operatorname{hom}_{\mathcal{V} \text {-smod }}(-, F)$ and $\operatorname{Hom}_{\mathcal{E}}(-, F(P))$ that evaluation on $P$ is fully faithful.

Next, we verify that evaluation is essentially surjective. Suppose $M \in{ }_{\mathcal{E}}$ smod. If follows from (35) that one may find a presentation of the form

$$
\mathcal{E} \otimes \mathbb{k}^{m_{2} \mid n_{2}} \stackrel{\psi}{\longrightarrow} \mathcal{E} \otimes \mathbb{k}^{m_{1} \mid n_{1}} \rightarrow M .
$$

Since evaluation on $P$ is fully faithful, there exists a natural transformation $\varphi$ : $h^{P} \otimes \mathbb{k}^{m_{2} \mid n_{2}} \rightarrow h^{P} \otimes \mathbb{k}^{m_{1} \mid n_{1}}$ which coincides with $\psi$ upon evaluation at $P$. Let us define a functor $F_{M}: \mathcal{V} \rightarrow \operatorname{svec}_{\mathbb{k}}$ by $F_{M}(X)=\operatorname{coker}\left(\varphi_{X}\right)$. Then $F_{M} \in \mathcal{V}$-smod is a functor whose evaluation at $P$ is isomorphic to $M$. Thus, evaluation at $P$ is essentially surjective.

\section{Appendix B. Superschemes And Supergroups}

We briefly recall the definitions and some basic properties of cosuperalgebras, superschemes and supergroups. For more details, see BrK1, BrK2 and the references therein.

B.1. Cosuperalgebras. A cosuperalgebra is a superspace $\mathcal{A}$ which is a coalgebra in the usual sense such that the comultiplication $\Delta_{\mathcal{A}}: \mathcal{A} \rightarrow \mathcal{A} \otimes \mathcal{A}$ and the counit $\epsilon$ : $\mathcal{A} \rightarrow \mathbb{k}$ are even linear maps. The notions of bisuperalgbra and Hopf cosuperalgebra can be defined similarly.

If $\mathcal{A}$ is a cosuperalgebra, a right $\mathcal{A}$-cosupermodule is a vector superspace $M$ together with a structure map $\Delta_{M}: M \rightarrow M \otimes \mathcal{A}$ which is an even linear map that makes $M$ into an ordinary comodule. Denote by $\operatorname{cosmod}_{\mathcal{A}}$ the category of all right $\mathcal{A}$-cosupermodules and $\mathcal{A}$-cosupermodule homomorphisms (which are just ordinary $\mathcal{A}$-comodule homormorphisms).

If $\mathcal{B}$ is a finite dimensional associative superalgebra, then multiplication in $\mathcal{B}$ gives an even linear map $m: \mathcal{B} \otimes \mathcal{B} \rightarrow \mathcal{B}$. Taking the dual of this map we obtain a linear map $\Delta=m^{\vee}: \mathcal{B}^{\vee} \rightarrow(\mathcal{B} \otimes \mathcal{B})^{\vee}=\mathcal{B}^{\vee} \otimes \mathcal{B}^{\vee}$, such that

$$
\langle\Delta(f), a \otimes b\rangle=(-1)^{|\Delta||f|}\langle f, a b\rangle=\langle f, a b\rangle
$$


(since $|\Delta|=|m|=0$ ), for $a, b \in \mathcal{B}, f \in \mathcal{B}^{\vee}$. This map $\Delta$ makes $\mathcal{B}^{\vee}$ into a cosuperalgebra.

Conversely, suppose that $\mathcal{A}$ is a finite dimensional cosuperalgebra. Then we make $\mathcal{A}^{\vee}$ into a superalgebra by defining the product $f g$ of $\mathbb{Z}_{2}$-homogeneous $f, g \in \mathcal{A}^{\vee}$ as

$$
\langle f g, a\rangle:=\left\langle f \otimes g, \Delta_{\mathcal{A}}(a)\right\rangle,
$$

for all $a \in \mathcal{A}$. Recall from BrK1 that there is an equivalence (in fact isomorphism) of categories between $\operatorname{cosmod}_{\mathcal{A}}$ and $\mathcal{A}^{\vee}$ smod.

Suppose $\mathcal{B}$ is an associative superalgebra. Then $\mathfrak{S}_{d}$ acts (on the right) on $\mathcal{B}^{\otimes d}$ via superalgebra automorphisms. Hence, $\Gamma^{d} \mathcal{B}=\left(\mathcal{B}^{\otimes d}\right)^{\mathfrak{S}_{d}}$ is also a superalgebra.

Now let $\mathcal{A}$ be a cosuperalgebra. Since $T^{*} \mathcal{A}$ is the free associative superalgebra generated by $\mathcal{A}$ (considered as a superspace), there is a unique superalgebra homomorphism

$$
\Delta: T^{*} \mathcal{A} \rightarrow T^{*} \mathcal{A} \otimes T^{*} \mathcal{A}
$$

such that $\Delta(a)=\Delta_{\mathcal{A}}(a)$ for all $a \in \mathcal{A}$, and $T^{*} \mathcal{A}$ is a cosuperalgebra with respect to this homomorphism. Similarly, since $S^{*} \mathcal{A}$ is a free commutative superalgebra, there exists a unique superalgebra homomorphism

$$
\widetilde{\Delta}: S^{*} \mathcal{A} \rightarrow S^{*} \mathcal{A} \otimes S^{*} \mathcal{A}
$$

such that $\widetilde{\Delta}(a)=\Delta_{\mathcal{A}}(a)$ for all $a \in \mathcal{A}$. (We note that a tensor product of commutative superalgebras is commutative.) The homomorphism $\widetilde{\Delta}$ makes $S^{*} \mathcal{A}$ into a cosuperalgebra.

One may check that we have

$$
\Delta\left(T^{d} \mathcal{A}\right) \subseteq T^{d} \mathcal{A} \otimes T^{d} \mathcal{A} \text { and } \quad \widetilde{\Delta}\left(S^{d} \mathcal{A}\right) \subseteq S^{d} \mathcal{A} \otimes S^{d} \mathcal{A} .
$$

Hence, both $T^{d} \mathcal{A}$ and $S^{d} \mathcal{A}$ may be considered as cosuperalgebras by restricting $\Delta$ and $\widetilde{\Delta}$ respectively.

Proposition B.1. Suppose $\mathcal{B}$ (resp. $\mathcal{A}$ ) is a finite dimensional associative superalgebra (resp. cosuperalgebra). Then we have the following isomorphisms of superalgebras.

(i) $\left(\mathcal{B}^{\vee}\right)^{\vee} \cong \mathcal{B}^{-}$, where $\mathcal{B}^{-}$is defined in Section 2 .

(ii) $\left(\mathcal{A}^{\vee}\right)^{\otimes d} \cong\left(\mathcal{A}^{\otimes d}\right)^{\vee}$.

(iii) $S^{d}\left(\mathcal{B}^{\vee}\right)^{\vee} \cong \Gamma^{d}\left(\mathcal{B}^{-}\right)$.

Proof. For (i) and (ii), the isomorphisms are given by the canonical even linear isomorphisms (1) and (22), respectively. It is then straightforward to check from the definitions that they are indeed superalgebra isomorphisms. For (iii), let $\mathcal{A}=\mathcal{B}^{\vee}$. Then one may check from parts (i) and (ii) that we have the following superalgebra isomorphisms:

$$
\begin{aligned}
S^{d}(\mathcal{A})^{\vee}=\left(\left(\mathcal{A}^{\otimes d}\right)_{\mathfrak{S}_{d}}\right)^{\vee} & \cong\left(\left(\mathcal{A}^{\otimes d}\right)^{\vee}\right)^{\mathfrak{S}_{d}} \\
& \cong\left(\left(\mathcal{A}^{\vee}\right)^{\otimes d}\right)^{\mathfrak{S}_{d}}=\Gamma^{d}\left(\mathcal{B}^{-}\right) .
\end{aligned}
$$

B.2. Superschemes. Let $\mathfrak{s a l \mathfrak { g } _ { \mathbb { k } }}$ denote the category of all commutative superalgebras and even homomorphisms. Also, let $\mathfrak{s s c h}_{\mathbb{k}}$ be the category of superschemes as in BrK2]. We may identify $\mathfrak{s s c h}_{\mathbb{k}}$ with a full subcategory of the category $\operatorname{Fct}\left(\mathfrak{s a l \mathfrak { g } _ { \mathbb { k } }}, \mathfrak{s e t} \mathfrak{s}\right)$ consisting of all functors from $\mathfrak{s a l} \mathfrak{g}_{\mathbb{k}}$ to $\mathfrak{s e t s}$. An affine superscheme is a representable functor $X=\operatorname{hom}_{\mathfrak{s a l} \mathfrak{g}_{\mathfrak{k}}}(\mathbb{k}[X],-)$, for some $\mathbb{k}[X] \in \mathfrak{s a l g}_{\mathbb{k}}$ which is called the coordinate ring of $X$. 
Given $M \in \operatorname{svec}_{\mathbb{k}}$, let $M_{a}: \mathfrak{s a l \mathfrak { g } _ { \mathbb { k } }} \rightarrow \mathfrak{s e t s}$ denote the functor defined by

$$
M_{a}(\mathcal{A})=(M \otimes \mathcal{A})_{0}
$$

for all $\mathcal{A} \in \mathfrak{s a l g}_{\mathfrak{k}}$. Then $M_{a}$ is an affine super scheme with coordinate ring given as follows. Suppose $N$ is an arbitrary superspace, not necessarily finite dimensional. Then we may identify $M^{\vee} \otimes N$ with $\operatorname{Hom}_{\mathbb{k}}(M, N)$ by setting

$$
(f \otimes w)(v)=(-1)^{|w||v|}\langle f, v\rangle w \quad\left(v \in M, w \in N, f \in M^{\vee}\right) .
$$

Then, for any $\mathcal{A} \in \mathfrak{s a l g}_{\mathfrak{k}}$, we have

$$
\begin{aligned}
M_{a}(\mathcal{A}) & =(M \otimes \mathcal{A})_{0}=\left(\left(M^{\vee}\right)^{\vee} \otimes \mathcal{A}\right)_{0} \\
& =\operatorname{Hom}_{\mathbb{k}}\left(M^{\vee}, \mathcal{A}\right)_{0}=\operatorname{hom}_{\mathfrak{s a l} \mathfrak{g}_{\mathfrak{k}}}\left(S^{*}\left(M^{\vee}\right), \mathcal{A}\right) .
\end{aligned}
$$

Hence $M_{a}$ is an affine superscheme with $\mathbb{k}\left[M_{a}\right]=S^{*}\left(M^{\vee}\right)$.

Now suppose $\mathcal{B}$ is an associative superalgebra. Let $V, W \in \mathcal{B} \operatorname{smod}$ and $\mathcal{A} \in \mathfrak{s a l}_{\mathbb{k}}$. Then it may be checked that formula (13) gives the following isomorphisms:

$$
\operatorname{Hom}_{\mathcal{B}}(V, W) \otimes \mathcal{A} \simeq \operatorname{Hom}_{B \otimes \mathcal{A}}(V \otimes \mathcal{A}, W \otimes \mathcal{A})
$$

where $\mathcal{A}$ is viewed as a supermodule over itself with respect to left multiplication.

Let $\operatorname{End}_{\mathcal{B}, V}$ denote the functor in $\operatorname{Fct}\left(\mathfrak{s a l} \mathfrak{g}_{\mathfrak{k}}, \mathfrak{s e t s}\right)$ such that $\operatorname{End}_{\mathcal{B}, V}(\mathcal{A})$ consists of the even $\mathcal{B} \otimes \mathcal{A}$-linear endomorphisms from $V \otimes \mathcal{A}$ to itself. Then, by identifying the left and right hand sides of (36), we see that $\operatorname{End}_{\mathcal{B}, V}=\left(\operatorname{End}_{\mathcal{B}}(V)\right)_{a}$. Hence, $\operatorname{End}_{\mathcal{B}, V}$ is an affine superscheme with

$$
\mathbb{k}\left[\operatorname{End}_{\mathcal{B}, V}\right]=S^{*}\left(\operatorname{End}_{\mathcal{B}}(V)^{\vee}\right) .
$$

Since $\operatorname{End}_{\mathcal{B}}(V)$ is a superalgebra, we may regard $\mathbb{k}\left[E n d_{\mathcal{B}, V}\right]$ as a cosuperalgebra via the map $\widetilde{\Delta}$ described above.

B.3. Supergroups. A supergroup is defined to be a functor $G$ from the category $\mathfrak{s a l} \mathfrak{g}_{\mathbb{k}}$ to the category $\mathfrak{g r o u p s}$. An algebraic supergroup is a supergroup $G$ which is also an affine superscheme, when viewed as a functor from $\mathfrak{s a l g}_{\mathfrak{k}}$ to $\mathfrak{s e t s}$, such that the coordinate ring $\mathbb{k}[G]$ is finitely generated. In this case, $\mathbb{k}[G]$ has a canonical structure of Hopf superalgebra. In particular, the comultiplication $\Delta: \mathbb{k}[G] \rightarrow$ $\mathbb{k}[G] \otimes \mathbb{k}[G]$ and counit $E: \mathbb{k}[G] \rightarrow G$ are defined, respectively, as the comorphisms of the multiplication and the unit of $G$.

Suppose $\mathcal{B}$ is an associative superalgebra and $V \in \mathcal{B}$ smod. Let $G L_{\mathcal{B}, V}$ denote the subfunctor of $E n d_{\mathcal{B}, V}$ such that $G L_{\mathcal{B}, V}(\mathcal{A})$ is the set of all even $\mathcal{B} \otimes \mathcal{A}$ linear automorphisms of $V \otimes \mathcal{A}$. Then $G L_{\mathcal{B}, V}$ is an algebraic supergroup, and $\mathbb{k}\left[\operatorname{End}_{\mathcal{B}, V}\right]=S^{*}\left(\operatorname{End}_{\mathcal{B}}(V)^{\vee}\right)$ is a subcosuperalgebra of $\mathbb{k}\left[G L_{\mathcal{B}, V}\right]$ with respect to the comultiplication $\widetilde{\Delta}$ defined above.

Example B.2. (i) Suppose $m, n$ are nonnegative integers. We use the notation

$$
M a t_{m \mid n}=E n d_{\mathbb{k}, \mathbb{k} m \mid n} \quad \text { and } \quad G L(m \mid n)=G L_{\mathbb{k}, \mathbb{k}^{m} \mid n} .
$$

If $\mathcal{A} \in \mathfrak{s a l}_{\mathfrak{g}_{\mathbb{k}}}$, then $\operatorname{Mat}_{m \mid n}(\mathcal{A})$ may be identified with the set of all matrices of the form

$$
\left(\begin{array}{ll}
A & B \\
C & D
\end{array}\right)
$$

where: $A$ is an $\mathcal{A}_{0}$-valued $m \times m$-matrix, $B$ is an $\mathcal{A}_{1}$-valued $m \times n$-matrix, $C$ is an $\mathcal{A}_{1}$-valued $n \times m$-matrix, and $D$ is an $\mathcal{A}_{0}$-valued $n \times n$-matrix. The matrix (37) corresponds to an even (resp. odd) linear operator if $B$ and $C$ 
(resp. $A$ and $D$ ) are both zero. From [L, Lemma 1.7.2 ], it follows that $G L(m \mid n, \mathcal{A})$ consists of all matrices (37) $\operatorname{such}$ that $\operatorname{det}(A) \operatorname{det}(D) \neq 0$.

Let $M=\mathbb{k}^{m \mid n}$. If $f \in \operatorname{End}_{\mathbb{k}}(M)$, we may decompose $f=f_{0}+f_{1}$, where $f_{0}$ is even and $f_{1}$ is odd. Let $\operatorname{det} \in S^{m+n}\left(\operatorname{End}_{\mathbb{k}}(M)^{\vee}\right)$ denote the element such that: for all $f \in \operatorname{End}_{\mathbb{k}}(M)$, $\operatorname{det}(f)=\operatorname{det}\left(\bar{f}_{0}\right)$, where the latter is the usual determinant of the induced linear operator $\bar{f}_{0}: \bar{M} \rightarrow \bar{M}$ of ordinary vector spaces. Then $G L(m \mid n)$ is an affine subsuperscheme of $M a t_{m \mid n}$, and $\mathbb{k}[G L(m \mid n)]$ is the localization of the coordinate ring $\mathbb{k}\left[M a t_{m \mid n}\right]$ at the element det.

(ii) Suppose $n$ is a nonnegative integer, and let $V=\mathcal{U}_{l}(1)^{\oplus n} \in \mathcal{C}(1)^{\text {smod. Then }}$ we write

$$
M a t_{n}=\operatorname{End}_{\mathcal{C}(1), V} \quad \text { and } \quad Q(n)=G L_{\mathcal{C}(1), V}
$$

From Example 2.6. it follows that $\operatorname{Mat}(\mathcal{A})$ may be identified with the set of matrices of the form

$$
\left(\begin{array}{cc}
S & S^{\prime} \\
-S^{\prime} & S
\end{array}\right),
$$

where $S$ (resp. $S^{\prime}$ ) is an $\mathcal{A}_{0}$-valued (resp. $\mathcal{A}_{1}$-valued) $n \times n$-matrix. The matrix (38) corresponds to an even (resp. odd) linear operator if $S^{\prime}=0$ (resp. $S=0)$.

Then $Q(n, \mathcal{A})$ consists of all invertible matrices of the form (38). We may define an element $\operatorname{det} \in \mathbb{k}[M a t]=S^{*}\left(\operatorname{End}_{\mathcal{C}(1)}(V)^{\vee}\right)$ in a way analogous to the previous example. It follows from $[\mathrm{BrK2}$ that $\mathbb{k}[Q(n)]$ is the localization of $\mathbb{k}[M a t]$ at det.

A representation of an algebraic supergroup $G$ is defined to be a natural transformation $\eta: G \rightarrow G L_{\mathbb{k}, M}$ for some $M \in \operatorname{svec}_{\mathbb{k}}$ such that $\eta_{\mathcal{A}}: G(\mathcal{A}) \rightarrow G L_{\mathbb{k}, M}(\mathcal{A})$ is a group homomorphism for each $\mathcal{A} \in \mathfrak{s a l} \mathfrak{g}_{\mathbb{k}}$. On the other hand, a $G$-supermodule is defined to be a right cosupermodule for the Hopf superalgebra $\mathbb{k}[G]$. The two notions of supermodule and representation are equivalent (cf. BrK2]). In particular, given a representation $\eta: G \rightarrow G L_{\mathcal{B}, M}$, there is a corresponding structure map

$$
\Delta_{M}: M \rightarrow M \otimes \mathbb{k}[G],
$$

making $M$ into a $G$-supermodule.

Definition B.3. Suppose $\mathcal{B}$ is a superalgebra and $V \in{ }_{\mathcal{B}}$ smod. Let $G=G L_{\mathcal{B}, V}$. Then we say that a representation $\eta: G \rightarrow G L_{\mathbb{k}, M}$ is polynomial if the image of the structure map $\Delta_{M}$ lies in $M \otimes \mathbb{k}\left[E n d_{\mathcal{B}, V}\right]$. We also let $\mathfrak{p o l}_{d}(G)$ denote the category of all homogeneous polynomial representations of degree $d$, which are defined to be the representations $M$ such that the image of $\Delta_{M}$ is contained in $\mathbb{k}\left[\operatorname{End}_{\mathcal{B}, V}\right]_{d}=S^{d}\left(\operatorname{End}_{\mathcal{B}}(V)^{\vee}\right)$. Notice that we have

$$
\mathfrak{p o l}_{d}(G)=\operatorname{cosmod}_{\mathbb{k}}\left[\operatorname{End}_{\mathcal{B}, V}\right]_{d}
$$

since $S^{d}\left(\operatorname{End}_{\mathcal{B}}(V)^{\vee}\right)$ is a subcosuperalgebra of $S^{*}\left(\operatorname{End}_{\mathcal{B}}(V)^{\vee}\right)$.

\section{ACKNOWLEDGMENTS}

The author wishes to thank Masaki Kashiwara and Myungho Kim, for many helpful conversations and suggestions, and Chris Drupieski, for useful feedback on an earlier version of this manuscript. The author would also like to thank the anonymous referee for helpful comments and suggestions. 


\section{REFERENCES}

[B] N. Bourbaki, Algebra. II. Chapters 4-7, Elements of Mathematics (Berlin), SpringerVerlag, Berlin, 1990. Translated from the French by P. M. Cohn and J. Howie. MR.1080964 (91h:00003)

[BrK1] Jonathan Brundan and Alexander Kleshchev, Projective representations of symmetric groups via Sergeev duality, Math. Z. 239 (2002), no. 1, 27-68, DOI 10.1007/s002090100282. MR.1879328 (2003b:20018)

[BrK2] Jonathan Brundan and Alexander Kleshchev, Modular representations of the supergroup $Q(n)$. I, J. Algebra 260 (2003), no. 1, 64-98, DOI 10.1016/S0021-8693(02)00620-8. Special issue celebrating the 80th birthday of Robert Steinberg. MR1973576 (2004f:20081)

[D] Stephen Donkin, Symmetric and exterior powers, linear source modules and representations of Schur superalgebras, Proc. London Math. Soc. (3) 83 (2001), no. 3, 647-680, DOI 10.1112/plms/83.3.647. MR 1851086 (2002i:20062)

[FS] Eric M. Friedlander and Andrei Suslin, Cohomology of finite group schemes over a field, Invent. Math. 127 (1997), no. 2, 209-270, DOI 10.1007/s002220050119. MR1427618 (98h:14055a)

[G] J. A. Green, Polynomial representations of $\mathrm{GL}_{n}$, With an appendix on Schensted correspondence and Littelmann paths by K. Erdmann, Green and M. Schocker. Second corrected and augmented edition, Lecture Notes in Mathematics, vol. 830, Springer, Berlin, 2007. MR2349209 (2009b:20084)

[HTY] J. Hong, A. Touzé, O. Yacobi. Polynomial functors and categorifications of Fock space, preprint: arXiv:1111.5317

[HY] Jiuzu Hong and Oded Yacobi, Polynomial functors and categorifications of Fock space II, Adv. Math. 237 (2013), 360-403, DOI 10.1016/j.aim.2013.01.004. MR3028582

[Jan] Jens Carsten Jantzen, Representations of algebraic groups, 2nd ed., Mathematical Surveys and Monographs, vol. 107, American Mathematical Society, Providence, RI, 2003. MR2015057(2004h:20061)

[Ke] Gregory Maxwell Kelly, Basic concepts of enriched category theory, London Mathematical Society Lecture Note Series, vol. 64, Cambridge University Press, Cambridge, 1982. MR651714(84e:18001)

[K] Alexander Kleshchev, Linear and projective representations of symmetric groups, Cambridge Tracts in Mathematics, vol. 163, Cambridge University Press, Cambridge, 2005. MR2165457 (2007b:20022)

[Kr] H. Krause, Koszul, Ringel and Serre duality for strict polynomial functors, Compos. Math. 149, (2013), no. 6, 996-1018. MR3077659

[L] D. A. Leĭtes, Introduction to the theory of supermanifolds, Uspekhi Mat. Nauk 35 (1980), no. 1(211), 3-57, 255 (Russian). MR565567 (81j:58003)

[Man] Yuri I. Manin, Gauge field theory and complex geometry, 2nd ed., Grundlehren der Mathematischen Wissenschaften [Fundamental Principles of Mathematical Sciences], vol. 289, Springer-Verlag, Berlin, 1997. Translated from the 1984 Russian original by N. Koblitz and J. R. King; With an appendix by Sergei Merkulov. MR.1632008 (99e:32001)

[P] Teimuraz Pirashvili, Introduction to functor homology, Rational representations, the Steenrod algebra and functor homology, Panor. Synthèses, vol. 16, Soc. Math. France, Paris, 2003, pp. 1-26 (English, with English and French summaries). MR2117526

[Ser] A. N. Sergeev, Tensor algebra of the identity representation as a module over the Lie superalgebras $\mathrm{Gl}(n, m)$ and $Q(n)$, Mat. Sb. (N.S.) 123(165) (1984), no. 3, 422-430 (Russian). MR735715 (85h:17010)

[T1] Antoine Touzé, Cohomology of classical algebraic groups from the functorial viewpoint, Adv. Math. 225 (2010), no. 1, 33-68, DOI 10.1016/j.aim.2010.02.014. MR2669348 (2012a:20074)

[T2] Antoine Touzé, Ringel duality and derivatives of non-additive functors, J. Pure Appl. Algebra 217 (2013), no. 9, 1642-1673, DOI 10.1016/j.jpaa.2012.12.007. MR.3042627

Department of Mathematics, Seoul National University, 599 Gwanak-ro, Gwanak-gu, SeOul 151-747, Korea

E-mail address: jdaxtell@snu.ac.kr 